\title{
Higher-order formulas of amplitude-dependent tune shift caused by a sextupole magnetic field distribution
}

\author{
Kouichi Soutome ${ }^{1,2, *}$ and Hitoshi Tanaka ${ }^{2}$ \\ ${ }^{1}$ Japan Synchrotron Radiation Research Institute, Kouto 1-1-1, Sayo, Hyogo 679-5198, Japan \\ ${ }^{2}$ RIKEN SPring-8 Center, Kouto 1-1-1, Sayo, Hyogo 679-5148, Japan
}

(Received 24 January 2017; published 13 June 2017)

\begin{abstract}
Nowadays, designs for ring-based light sources use multibend lattices for achieving a very small emittance of around 100 pmrad. In this type of storage ring, the chromaticity correcting sextupoles generally have greater strengths than those used in typical third-generation light sources. Therefore, controlling lattice nonlinearity such as amplitude-dependent tune shift (ADTS) is important for enabling stable operations and smooth beam commissioning. As the strength of the sextupoles increases, their higher-order terms contribute significantly to ADTS, rendering well-known lowest-order formulas inadequate for describing tune variations at large horizontal amplitudes. In response, we have derived explicit expressions of ADTS up to the fourth order in sextupole strength based on the canonical perturbation theory, assuming that the amplitude of a vertical betatron oscillation is smaller compared with the horizontal one. The new formulas express the horizontal and vertical betatron tune variations as functions of the action variables: $J_{x}$ and $J_{y}$ up to $O\left(J_{x}^{2}\right)$ and $O\left(J_{y}\right)$. The derived formulas were applied to a five-bend achromat lattice designed for the SPring- 8 upgrade. By comparing the calculated results with the tracking simulations, we found that (1) the formulas accurately express ADTS around a horizontal amplitude of $\sim 10 \mathrm{~mm}$ and (2) the nonlinear terms of the fourth order in sextupole strength govern the behaviors of circulating electrons at large horizontal amplitudes. In this paper, we present explicit expressions of fourth-order formulas of ADTS and provide some examples to illustrate their effectiveness.
\end{abstract}

DOI: 10.1103/PhysRevAccelBeams.20.064001

\section{INTRODUCTION}

Optimizing lattice nonlinearity presents a major challenge for designing a storage ring with strong sextupole magnets. The amplitude dependence of betatron tunes is one of the key parameters in the optimization procedure for avoiding unstable beam motions resulting from harmful resonance lines by choosing a proper working point and by suppressing tune variations with amplitudes. The lowestorder formulas of the amplitude-dependent tune shifts (ADTS) are well known [1,2] and are usually incorporated in an objective function of nonlinearity optimization. However, for a ring with very strong sextupole magnets, the lowest-order formulas are no longer effective for describing tune variations at large amplitudes near a border of dynamic aperture because of the dominant contributions from the higher-order terms.

A good example where the higher-order terms govern beam behavior at large amplitudes is the type of multibend achromat lattices adopted in many light source facilities to

\footnotetext{
*soutome@spring8.or.jp
}

Published by the American Physical Society under the terms of the Creative Commons Attribution 4.0 International license. Further distribution of this work must maintain attribution to the author(s) and the published article's title, journal citation, and DOI. achieve extremely small emittance [3]. At SPring-8, a fivebend achromat (5BA) lattice was selected for the basic structure of the storage ring for the performance upgrade [4]. The maximum strength of the chromaticity-correcting sextupoles is about six times larger than that for the present ring, which uses a double-bend lattice for its unit structure. Our approach to sextupole optimization is based on an analytical model with ADTS coefficients incorporated in the objective function [5]. In optimization procedures, we first used the lowest-order formulas of ADTS to suppress tune variations to obtain larger dynamic apertures. However, after some calculations we found that the lowest-order formulas are insufficient to describe tune variations at large horizontal amplitudes. Tracking simulations consistently indicate that the higher-order terms in sextupole strength govern the behavior of electrons at large amplitudes. Hence we need to estimate higher order terms by other means.

In the 1990s, techniques were developed for computing a map that transfers initial coordinates at some reference position to final coordinates. These techniques provided access to higher-order effects up to a desired order [6-10] numerically. Maps could be calculated using Lie transformations or truncated Taylor series with algebraic techniques. The maps obtained could be used for faster tracking instead of the conventional element-by-element approach. Also, by casting a map into the so-called normal form [11], interesting physical quantities such as nonlinear 
chromaticities and amplitude-dependent tune shifts could be extracted up to quite a high order. The map method is therefore one of the standard and powerful tools used for studying nonlinear beam dynamics, offering great benefits for analyzing nonlinear beam behavior: once a magnetic lattice is given and a map is calculated, one can easily derive higher-order coefficients numerically and predict how the beam will behave at large amplitudes or large momentum deviations.

In design work, however, we usually start by trying to understand a mechanism (or beam physics) that dominates the nonlinear behavior of the beam. Needless to say, it is better to understand the underlying mechanisms that determine higher-order coefficients of ADTS in order to proceed efficiently with nonlinear lattice design. From this viewpoint, we insisted on an analytical method and tried to derive explicit expressions of the higher-order coefficients of ADTS, though the optimization itself would be possible in a completely numerical manner by incorporating the map method in the optimization algorithm with proper constraints. We also point out that when one tries to find an optimum set of sextupole strengths in a numerical manner, a procedure for map calculation and tracking is needed for each trial set of sextupoles. The purpose of our paper is to offer another convenient tool for studying and mitigating lattice nonlinearity. For example, for the design of a basic lattice of a unit cell having a moderate number of sextupole magnets, our formulas [Eqs. (96)-(98)] are applicable for fast evaluation of the objective function in nonlinear optimization.

However, as one can easily imagine, it is not an easy task to extract high-order lattice nonlinearities in an analytic form. In fact, the explicit expressions of ADTS of the fourth-order or higher in sextupole strength are, to the authors' knowledge, not found in literature. This is because the number of terms in canonical perturbation treatments increases rapidly as we proceed to higher orders. For our goal of optimizing sextupole strengths for a 5BA lattice, we need to describe the behavior of both the horizontal and the vertical tunes correctly at large horizontal amplitudes [12]. Hence, we assumed that the amplitude of the vertical betatron oscillation is smaller compared with the horizontal one and neglected terms of $O\left(J_{y}^{2}\right)$, where $J_{y}$ is the action variable of the vertical oscillation, in calculating the Hamiltonian of the fourth order in sextupole strength. Our assumption is valid in most practical cases for discussing the beam injections and betatron oscillations caused by electron-electron scattering, in which tune variations depending on the horizontal amplitude govern stability in motion. This assumption greatly reduces the number of terms that we have to treat and allows the analytical description of explicit expressions of higherorder formulas of ADTS.

In Sec. II, we briefly review application of the canonical perturbation theory to storage rings with a sextupole magnetic field distribution. The Hamiltonian is expressed by a sum of terms having a power series of sextupole field strength $\lambda(s)$. We keep terms up to $\lambda^{4}$ since the next-order contributions to the well-known ADTS formulas come from the Hamiltonian terms of $O\left(\lambda^{4}\right)$. Details of higherorder perturbation treatments are described in Sec. III. As mentioned above, we assume that the amplitude of the vertical betatron oscillation is smaller compared with the horizontal one to simplify the canonical perturbation treatments. In Sec. IV, we apply the formulas to a five-bend achromat lattice for the SPring-8 upgrade and check their validity by comparing our analytical calculations with tracking simulations. Finally, we summarize our results in the last section.

\section{CANONICAL PERTURBATION THEORY}

\section{A. Hamiltonian for a ring with a sextupole magnetic field distribution}

We first review the canonical perturbation theory [13-15]. For storage rings with a sextupole magnetic field distribution $B^{\prime \prime}(s)$, the transverse motion of electrons $\left(x, p_{x}, y, p_{y}\right)$ is described by the Hamiltonian

$$
H=H_{0}+V,
$$

where

$$
H_{0}=\frac{p_{x}^{2}}{2}+\frac{p_{y}^{2}}{2}+\frac{1}{2}\left(g(s)+\frac{1}{\rho^{2}(s)}\right) x^{2}-\frac{1}{2} g(s) y^{2}
$$

is for the linear betatron oscillation and

$$
V=\frac{\lambda(s)}{6}\left(x^{3}-3 x y^{2}\right)
$$

is the sextupole field potential with

$$
\lambda(s) \equiv \frac{B^{\prime \prime}(s)}{[B \rho]} .
$$

The $g(s)$ is the quadrupole field potential, $\rho(s)$ is the bending radius, and $[B \rho]$ is the magnetic rigidity. By using a generating function

$$
\begin{aligned}
F\left(x, \phi_{x}, y, \phi_{y}, s\right)= & -\frac{x^{2}}{2 \beta_{x}(s)}\left(\tan \phi_{x}-\frac{\beta_{x}^{\prime}(s)}{2}\right) \\
& -\frac{y^{2}}{2 \beta_{y}(s)}\left(\tan \phi_{y}-\frac{\beta_{y}^{\prime}(s)}{2}\right),
\end{aligned}
$$

where $\beta_{x}$ and $\beta_{y}$ are the horizontal and the vertical betatron functions, respectively, we perform a canonical transformation from the coordinate $\left(x, p_{x}, y, p_{y}\right)$ to the actionangle one $\left(\phi_{x}, I_{x}, \phi_{y}, I_{y}\right)$ : 


$$
\begin{aligned}
x & =\sqrt{2 I_{x} \beta_{x}(s)} \cos \phi_{x}, \\
p_{x} & =-\sqrt{2 I_{x} / \beta_{x}(s)}\left(\sin \phi_{x}-\frac{\beta_{x}^{\prime}(s)}{2} \cos \phi_{x}\right), \\
y & =\sqrt{2 I_{y} \beta_{y}(s)} \cos \phi_{y},
\end{aligned}
$$

$$
\begin{array}{r}
p_{y}=-\sqrt{2 I_{y} / \beta_{y}(s)}\left(\sin \phi_{y}-\frac{\beta_{y}^{\prime}(s)}{2} \cos \phi_{y}\right), \\
H\left(\phi_{x}, I_{x}, \phi_{y}, I_{y}, s\right)=H_{0}\left(I_{x}, I_{y}, s\right)+V\left(\phi_{x}, I_{x}, \phi_{y}, I_{y}, s\right), \\
H_{0}\left(I_{x}, I_{y}, s\right)=\frac{I_{x}}{\beta_{x}(s)}+\frac{I_{y}}{\beta_{y}(s)},
\end{array}
$$

By following the treatment of the canonical perturbation theory with $\lambda$ being a perturbation source, we can find a canonical transformation from $\left(\phi_{x}, I_{x}, \phi_{y}, I_{y}\right)$ to $\left(\varphi_{x}, J_{x}\right.$, $\left.\varphi_{y}, J_{y}\right)$, where a new Hamiltonian $\tilde{H}$ is written by only $J_{x}$ and $J_{y}$ up to some order in $\lambda$. To do this, we first write a generating function $G$ in the following form:

$$
\begin{aligned}
G\left(\phi_{x}, J_{x}, \phi_{y}, J_{y}, s\right)= & \phi_{x} J_{x}+\phi_{y} J_{y} \\
& +\sum_{n=1}^{\infty} G^{(n)}\left(\phi_{x}, J_{x}, \phi_{y}, J_{y}, s\right),
\end{aligned}
$$

where $G^{(n)}$ is a contribution of $O\left(\lambda^{n}\right)$. The transformation is then carried out by

$$
\begin{gathered}
I_{x}=\frac{\partial G}{\partial \phi_{x}}=J_{x}+\sum_{n=1}^{\infty} \frac{\partial G^{(n)}\left(\phi_{x}, J_{x}, \phi_{y}, J_{y}, s\right)}{\partial \phi_{x}}, \\
\varphi_{x}=\frac{\partial G}{\partial J_{x}}=\phi_{x}+\sum_{n=1}^{\infty} \frac{\partial G^{(n)}\left(\phi_{x}, J_{x}, \phi_{y}, J_{y}, s\right)}{\partial J_{x}}, \\
I_{y}=\frac{\partial G}{\partial \phi_{y}}=J_{y}+\sum_{n=1}^{\infty} \frac{\partial G^{(n)}\left(\phi_{x}, J_{x}, \phi_{y}, J_{y}, s\right)}{\partial \phi_{y}}, \\
\varphi_{y}=\frac{\partial G}{\partial J_{y}}=\phi_{y}+\sum_{n=1}^{\infty} \frac{\partial G^{(n)}\left(\phi_{x}, J_{x}, \phi_{y}, J_{y}, s\right)}{\partial J_{y}}, \\
\tilde{H}=\frac{H+\frac{\partial G}{\partial s}=}{H_{0}\left(I_{x}, I_{y}, s\right)+V\left(\phi_{x}, I_{x}, \phi_{y}, I_{y}, s\right)} \\
+\sum_{n=1}^{\infty} \frac{\partial G^{(n)}\left(\phi_{x}, J_{x}, \phi_{y}, J_{y}, s\right)}{\partial s} .
\end{gathered}
$$

By using Eqs. (14) and (16) in the first and second terms of Eq. (18) and carrying out a Taylor expansion in $\left(I_{x}, I_{y}\right)$ at $\left(J_{x}, J_{y}\right)$ up to the fourth order in sextupole strength, we have

$$
\begin{aligned}
& \tilde{H}=\tilde{H}^{(0)}+\tilde{H}^{(1)}+\tilde{H}^{(2)}+\tilde{H}^{(3)}+\tilde{H}^{(4)}+O\left(\lambda^{5}\right) \\
& \tilde{H}^{(0)} \equiv \frac{J_{x}}{\beta_{x}}+\frac{J_{y}}{\beta_{y}}, \\
& \tilde{H}^{(1)} \equiv V+\frac{1}{\beta_{x}} \frac{\partial G^{(1)}}{\partial \phi_{x}}+\frac{1}{\beta_{y}} \frac{\partial G^{(1)}}{\partial \phi_{y}}+\frac{\partial G^{(1)}}{\partial s} \\
& \tilde{H}^{(2)} \equiv \frac{1}{\beta_{x}} \frac{\partial G^{(2)}}{\partial \phi_{x}}+\frac{1}{\beta_{y}} \frac{\partial G^{(2)}}{\partial \phi_{y}}+\frac{\partial V}{\partial J_{x}} \frac{\partial G^{(1)}}{\partial \phi_{x}} \\
&+\frac{\partial V}{\partial J_{y}} \frac{\partial G^{(1)}}{\partial \phi_{y}}+\frac{\partial G^{(2)}}{\partial s}, \\
& \tilde{H}^{(3)} \equiv \frac{1}{\beta_{x}} \frac{\partial G^{(3)}}{\partial \phi_{x}}+\frac{1}{\beta_{y}} \frac{\partial G^{(3)}}{\partial \phi_{y}}+\frac{\partial V}{\partial J_{x}} \frac{\partial G^{(2)}}{\partial \phi_{x}}+\frac{\partial V}{\partial J_{y}} \frac{\partial G^{(2)}}{\partial \phi_{y}} \\
&+\frac{1}{2} \frac{\partial^{2} V}{\partial J_{x}^{2}}\left(\frac{\partial G^{(1)}}{\partial \phi_{x}}\right)^{2}+\frac{\partial^{2} V}{\partial J_{x} \partial J_{y}} \frac{\partial G^{(1)}}{\partial \phi_{x}} \frac{\partial G^{(1)}}{\partial \phi_{y}}+\frac{\partial G^{(3)}}{\partial s}, \\
&+\frac{1}{2} \frac{\partial^{3} V}{\partial J_{x}^{2} \partial J_{y}}\left(\frac{\partial G^{(1)}}{\partial \phi_{x}}\right)^{2} \frac{\partial G^{(1)}}{\partial \phi_{y}}+\frac{\partial G^{(4)}}{\partial s} . \\
& \tilde{H}^{(4)} \equiv \frac{1}{\beta_{x}} \frac{\partial G^{(4)}}{\partial \phi_{x}}+\frac{1}{\beta_{y}} \frac{\partial G^{(4)}}{\partial \phi_{y}}+\frac{\partial V}{\partial J_{x}} \frac{\partial G^{(3)}}{\partial \phi_{x}}+\frac{\partial V}{\partial J_{y}} \frac{\partial G^{(3)}}{\partial \phi_{y}} \\
&+\frac{\partial^{2} V}{\partial J_{x}^{2}} \frac{\partial G^{(1)}}{\partial \phi_{x}} \frac{\partial G^{(2)}}{\partial \phi_{x}}+\frac{\partial^{2} V}{\partial J_{x} \partial J_{y}} \frac{\partial G^{(1)}}{\partial \phi_{x}} \frac{\partial G^{(2)}}{\partial \phi_{y}} \\
& \frac{\partial G^{(2)}}{\partial \phi_{y}}+\frac{1}{\partial \phi^{3} V}\left(\frac{\partial G^{(1)}}{\partial \phi_{x}}\right)^{3} \\
&
\end{aligned}
$$

The term $\tilde{H}^{(n)}$ represents a quantity of $O\left(\lambda^{n}\right)$ and the argument of each function on the right-hand side is $\left(\phi_{x}, J_{x}, \phi_{y}, J_{y}, s\right)$. We see that the expressions of $\tilde{H}^{(3)}$ and $\tilde{H}^{(4)}$ are asymmetric with respect to $x$ and $y$ because 
$\partial^{k} V\left(\phi_{x}, J_{x}, \phi_{y}, J_{y}, s\right) / \partial J_{y}^{k}=0$ for $k \geq 2$. The $n$ th-order generating function $G^{(n)}$ is determined to satisfy

$$
\tilde{H}^{(n)}-\left\langle\tilde{H}^{(n)}\right\rangle=0,
$$

where $\langle A\rangle$ means the average of $A$ over $\phi_{x}$ and $\phi_{y}$. Then, after calculating generating functions up to the $N$ th order by solving Eq. (25) step by step, the Hamiltonian (19) is approximated as the following autonomous form with the $N$ th order in sextupole strength:

$$
\begin{aligned}
\tilde{H} & =\tilde{H}^{(0)}+\sum_{n=1}^{\infty}\left\{\tilde{H}^{(n)}-\left\langle\tilde{H}^{(n)}\right\rangle\right\}+\sum_{n=1}^{\infty}\left\langle\tilde{H}^{(n)}\right\rangle \\
& =\tilde{H}^{(0)}+\sum_{n=1}^{N}\left\langle\tilde{H}^{(n)}\right\rangle+O\left(\lambda^{N+1}\right) .
\end{aligned}
$$

The betatron tune is calculated by

$$
\tilde{\nu}_{z}=\frac{1}{2 \pi} \int_{0}^{C} d s \frac{\partial \tilde{H}}{\partial J_{z}} \quad(z=x, y),
$$

where $C$ is the circumference.

\section{B. Lowest-order perturbation}

The first-order generating function $G^{(1)}$ is obtained by solving Eq. (25) with $n=1$ :

$$
\begin{aligned}
& \frac{1}{\beta_{x}} \frac{\partial G^{(1)}}{\partial \phi_{x}}+\frac{1}{\beta_{y}} \frac{\partial G^{(1)}}{\partial \phi_{y}}+\frac{\partial G^{(1)}}{\partial s}=-V^{(1)}, \\
& V^{(1)} \equiv V-\left\langle V+\frac{1}{\beta_{x}} \frac{\partial G^{(1)}}{\partial \phi_{x}}+\frac{1}{\beta_{y}} \frac{\partial G^{(1)}}{\partial \phi_{y}}+\frac{\partial G^{(1)}}{\partial s}\right\rangle .
\end{aligned}
$$

From the periodicity of $G^{(1)}$, we have the following conditions:

$$
\left\langle\frac{\partial G^{(1)}}{\partial \phi_{x}}\right\rangle=\left\langle\frac{\partial G^{(1)}}{\partial \phi_{y}}\right\rangle=0 \quad \text { and } \quad\left\langle\frac{\partial G^{(1)}}{\partial s}\right\rangle=0 .
$$

These relations can be confirmed by Eqs. (37) and (42) given below. Equation (12) gives $\langle V\rangle=0$ and hence

$$
\begin{aligned}
V^{(1)}= & V\left(\phi_{x}, J_{x}, \phi_{y}, J_{y}, s\right) \\
= & \frac{2}{3} J_{x}^{3 / 2} A_{1}(s)\left(e_{3,0}+e_{-3,0}+3 e_{1,0}+3 e_{-1,0}\right) \\
& -2 J_{x}^{1 / 2} J_{y} A_{2}(s)\left(2 e_{1,0}+2 e_{-1,0}+e_{1,2}\right. \\
& \left.+e_{-1,-2}+e_{1,-2}+e_{-1,2}\right) \\
\equiv & \sum_{m, n=-\infty}^{+\infty} v_{m, n}^{(1)} e_{m, n},
\end{aligned}
$$

$$
A_{1}(s) \equiv \frac{1}{8 \sqrt{2}} \lambda(s) \beta_{x}^{3 / 2}(s)
$$

$$
\begin{aligned}
A_{2}(s) & \equiv \frac{1}{8 \sqrt{2}} \lambda(s) \beta_{x}^{1 / 2}(s) \beta_{y}(s), \\
e_{m, n} & \equiv e^{i\left(m \phi_{x}+n \phi_{y}\right)}
\end{aligned}
$$

There are eight nonzero terms of $v_{m, n}^{(1)}$ :

$$
\begin{aligned}
v_{3,0}^{(1)} & =v_{-3,0}^{(1)}=\frac{2}{3} J_{x}^{3 / 2} A_{1}(s), \\
v_{1,0}^{(1)} & =v_{-1,0}^{(1)}=2 J_{x}^{3 / 2} A_{1}(s)-4 J_{x}^{1 / 2} J_{y} A_{2}(s), \\
v_{1,2}^{(1)} & =v_{-1,-2}^{(1)}=v_{1,-2}^{(1)}=v_{-1,2}^{(1)}=-2 J_{x}^{1 / 2} J_{y} A_{2}(s), \\
v_{m, n}^{(1)} & =0 \quad \text { for other }(m, n) .
\end{aligned}
$$

Equation (28) is now written as

$$
\left\{\frac{1}{\beta_{x}} \frac{\partial}{\partial \phi_{x}}+\frac{1}{\beta_{y}} \frac{\partial}{\partial \phi_{y}}+\frac{\partial}{\partial s}\right\} G^{(1)}=-\sum_{m, n} v_{m, n}^{(1)} e_{m, n}
$$

and a periodic solution to this equation is given by

$G^{(1)}=\sum_{m, n=-\infty}^{+\infty} g_{m, n}^{(1)} e_{m, n}$

$$
\begin{aligned}
g_{m, n}^{(1)} \equiv & \frac{i}{2 \sin \pi\left(m \nu_{x}+n \nu_{y}\right)} \\
& \times \int_{s}^{s+C} d s^{\prime} v_{m, n}^{(1)}\left(J_{x}, J_{y}, s^{\prime}\right) e^{i m \Psi_{x}\left(s^{\prime}, s\right)+i n \Psi_{y}\left(s^{\prime}, s\right)},
\end{aligned}
$$

$\Psi_{z}\left(s^{\prime}, s\right) \equiv \psi_{z}\left(s^{\prime}\right)-\psi_{z}(s)-\pi \nu_{z} \quad(z=x, y)$,

$$
\begin{gathered}
\psi_{z}(s) \equiv \int_{0}^{s} \frac{d s^{\prime}}{\beta_{z}\left(s^{\prime}\right)}, \\
\nu_{z}=\frac{\psi_{z}(C)}{2 \pi} .
\end{gathered}
$$

By using Eq. (35) we have the following expressions for $g_{m, n}^{(1)}$ : 


$$
\begin{aligned}
g_{3,0}^{(1)} & =g_{-3,0}^{(1) *}=\frac{i}{3} J_{x}^{3 / 2} B_{2}(s) \\
g_{1,0}^{(1)} & =g_{-1,0}^{(1) *}=i J_{x}^{3 / 2} B_{1}(s)-2 i J_{x}^{1 / 2} J_{y} B_{3}(s), \\
g_{1,2}^{(1)} & =g_{-1,-2}^{(1) *}=-i J_{x}^{1 / 2} J_{y} B_{4}(s) \\
g_{1,-2}^{(1)} & =g_{-1,2}^{(1) *}=-i J_{x}^{1 / 2} J_{y} B_{5}(s) \\
g_{m, n}^{(1)} & =0 \quad \text { for other }(m, n)
\end{aligned}
$$

where we have defined

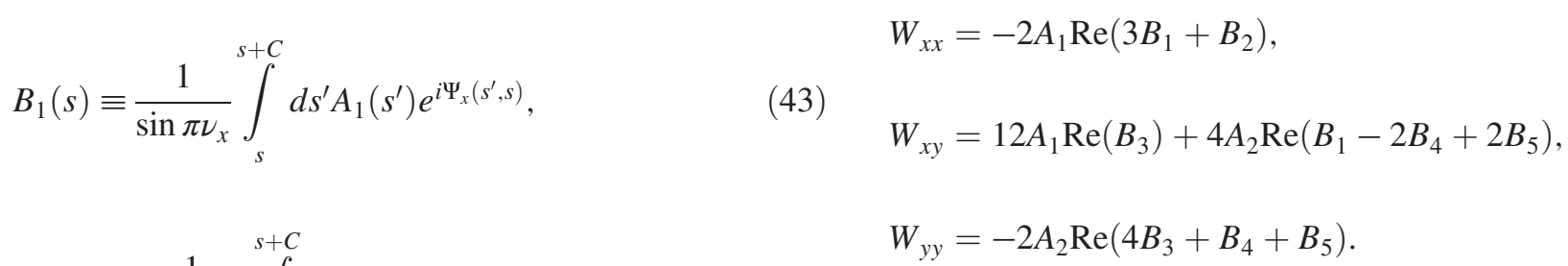$$
B_{2}(s) \equiv \frac{1}{\sin 3 \pi \nu_{x}} \int_{s}^{s+C} d s^{\prime} A_{1}\left(s^{\prime}\right) e^{i 3 \Psi_{x}\left(s^{\prime}, s\right)},
$$$$
B_{3}(s) \equiv \frac{1}{\sin \pi \nu_{x}} \int_{s}^{s+C} d s^{\prime} A_{2}\left(s^{\prime}\right) e^{i \Psi_{x}\left(s^{\prime}, s\right)}
$$$$
B_{4}(s) \equiv \frac{1}{\sin \pi\left(\nu_{x}+2 \nu_{y}\right)} \int_{s}^{s+C} d s^{\prime} A_{2}\left(s^{\prime}\right) e^{i\left\{\Psi_{x}\left(s^{\prime}, s\right)+2 \Psi_{y}\left(s^{\prime}, s\right)\right\}},
$$$$
B_{5}(s) \equiv \frac{1}{\sin \pi\left(\nu_{x}-2 \nu_{y}\right)} \int_{s}^{s+C} d s^{\prime} A_{2}\left(s^{\prime}\right) e^{i\left\{\Psi_{x}\left(s^{\prime}, s\right)-2 \Psi_{y}\left(s^{\prime}, s\right)\right\}} .
$$

We then have

$$
\left\langle\tilde{H}^{(1)}\right\rangle=\left\langle V+\frac{1}{\beta_{x}} \frac{\partial G^{(1)}}{\partial \phi_{x}}+\frac{1}{\beta_{y}} \frac{\partial G^{(1)}}{\partial \phi_{y}}+\frac{\partial G^{(1)}}{\partial s}\right\rangle=0,
$$

and, as seen from Eqs. (26) and (27), the betatron tune is not shifted by the perturbation of $O\left(\lambda^{1}\right)$.

With the expression (37) for $G^{(1)}$, we can evaluate the average of the next-order term of the Hamiltonian (22):

$$
\left\langle\tilde{H}^{(2)}\right\rangle=\left\langle\frac{\partial V}{\partial J_{x}} \frac{\partial G^{(1)}}{\partial \phi_{x}}+\frac{\partial V}{\partial J_{y}} \frac{\partial G^{(1)}}{\partial \phi_{y}}\right\rangle,
$$

where we have used the fact that

$$
\left\langle\frac{\partial G^{(2)}}{\partial \phi_{x}}\right\rangle=\left\langle\frac{\partial G^{(2)}}{\partial \phi_{y}}\right\rangle=0 \quad \text { and } \quad\left\langle\frac{\partial G^{(2)}}{\partial s}\right\rangle=0,
$$

These relations hold due to the periodicity of $G^{(2)}$ as seen in the first-order case. By substituting Eqs. (31) and (37) into Eq. (49) and collecting angle-independent terms proportional to $e_{0,0}$, we obtain

$\left\langle\tilde{H}^{(2)}\right\rangle=W_{x x} J_{x}^{2}+W_{x y} J_{x} J_{y}+W_{y y} J_{y}^{2}$,

Here, we have used the following relations:

$$
\begin{aligned}
e_{m_{1}, n_{1}} e_{m_{2}, n_{2}} & =e_{m_{1}+m_{2}, n_{1}+n_{2}}, \\
e_{-m,-n} & =e_{m, n}^{*}, \\
\frac{\partial}{\partial \phi_{x}} e_{m, n} & =i m e_{m, n}, \\
\frac{\partial}{\partial \phi_{y}} e_{m, n} & =i n e_{m, n} .
\end{aligned}
$$

From Eq. (27) with $\tilde{H}=\tilde{H}^{(0)}+\left\langle\tilde{H}^{(2)}\right\rangle$ we have the following expressions for the betatron tune:

$\tilde{\nu}_{x}=\frac{1}{2 \pi} \int_{0}^{C} \frac{d s}{\beta_{x}}+\frac{1}{2 \pi} \int_{0}^{C} 2 W_{x x}(s) d s J_{x}+\frac{1}{2 \pi} \int_{0}^{C} W_{x y}(s) d s J_{y}$,

$\tilde{\nu}_{y}=\frac{1}{2 \pi} \int_{0}^{C} \frac{d s}{\beta_{y}}+\frac{1}{2 \pi} \int_{0}^{C} W_{x y}(s) d s J_{x}+\frac{1}{2 \pi} \int_{0}^{C} 2 W_{y y}(s) d s J_{y}$.

It is apparent that these are equivalent to the well-known lowest-order formulas of ADTS [1,2]:

$$
\begin{aligned}
& \tilde{\nu}_{x}=\nu_{x}+\alpha_{x x} J_{x}+\alpha_{x y} J_{y}, \\
& \tilde{\nu}_{y}=\nu_{y}+\alpha_{y x} J_{x}+\alpha_{y y} J_{y},
\end{aligned}
$$




$$
\begin{aligned}
\alpha_{x x}= & -\frac{1}{64 \pi} \int_{0}^{C} d s \lambda(s) \beta_{x}^{3 / 2}(s) \int_{s}^{s+C} d s^{\prime} \lambda\left(s^{\prime}\right) \beta_{x}^{3 / 2}\left(s^{\prime}\right)\left[\frac{3 \cos \Psi_{x}\left(s^{\prime}, s\right)}{\sin \pi \nu_{x}}+\frac{\cos 3 \Psi_{x}\left(s^{\prime}, s\right)}{\sin 3 \pi \nu_{x}}\right], \\
\alpha_{x y}= & \alpha_{y x}=\frac{3}{64 \pi} \int_{0}^{C} d s \lambda(s) \beta_{x}^{3 / 2}(s) \int_{s}^{s+C} d s^{\prime} \lambda\left(s^{\prime}\right) \beta_{x}^{1 / 2}\left(s^{\prime}\right) \beta_{y}\left(s^{\prime}\right) \frac{\cos \Psi_{x}\left(s^{\prime}, s\right)}{\sin \pi \nu_{x}}+\frac{1}{64 \pi} \int_{0}^{C} d s \lambda(s) \beta_{x}^{1 / 2}(s) \beta_{y}(s) \\
& \times \int_{s}^{s+C} d s^{\prime} \lambda\left(s^{\prime}\right) \beta_{x}^{3 / 2}\left(s^{\prime}\right) \frac{\cos \Psi_{x}\left(s^{\prime}, s\right)}{\sin \pi \nu_{x}}-\frac{1}{32 \pi} \int_{0}^{C} d s \lambda(s) \beta_{x}^{1 / 2}(s) \beta_{y}(s) \int_{s} d s^{\prime} \lambda\left(s^{\prime}\right) \beta_{x}^{1 / 2}\left(s^{\prime}\right) \beta_{y}\left(s^{\prime}\right) \\
& \times\left[\frac{\cos \left\{\Psi_{x}\left(s^{\prime}, s\right)+2 \Psi_{y}\left(s^{\prime}, s\right)\right\}}{\sin \pi\left(\nu_{x}+2 \nu_{y}\right)}-\frac{\cos \left\{\Psi_{x}\left(s^{\prime}, s\right)-2 \Psi_{y}\left(s^{\prime}, s\right)\right\}}{\sin \pi\left(\nu_{x}-2 \nu_{y}\right)}\right], \\
\alpha_{y y}= & -\frac{1}{64 \pi} \int_{0}^{C} d s \lambda(s) \beta_{x}^{1 / 2}(s) \beta_{y}(s) \int_{s} d s^{\prime} \lambda\left(s^{\prime}\right) \beta_{x}^{1 / 2}\left(s^{\prime}\right) \beta_{y}\left(s^{\prime}\right) \\
& \left.\times 4 \frac{\cos \Psi_{x}\left(s^{\prime}, s\right)}{\sin \pi \nu_{x}}+\frac{\cos \left\{\Psi_{x}\left(s^{\prime}, s\right)+2 \Psi_{y}\left(s^{\prime}, s\right)\right\}}{\sin \pi\left(\nu_{x}+2 \nu_{y}\right)}+\frac{\cos \left\{\Psi_{x}\left(s^{\prime}, s\right)-2 \Psi_{y}\left(s^{\prime}, s\right)\right\}}{\sin \pi\left(\nu_{x}-2 \nu_{y}\right)}\right] .
\end{aligned}
$$

\section{HIGHER-ORDER FORMULAS}

\section{A. Assumptions for a small vertical oscillation amplitude}

The procedure of canonical perturbation treatment itself is well established. In theory, it is straightforward to perform treatments up to any order in sextupole strength. In practice, however, when we move beyond the lowestorder transformation we soon encounter a rapid increase in the number of terms that we have to manipulate [16]. Moreover, as is well known [13] and we will see later, the next-order contributions to ADTS come from the fourthorder Hamiltonian $\left\langle\tilde{H}^{(4)}\right\rangle$ since the third-order Hamiltonian $\left\langle\tilde{H}^{(3)}\right\rangle$ vanishes. This means that in order to obtain explicit expressions of the next-order formula of ADTS, the perturbation procedure must be repeated twice more, which is practically impossible.

Therefore we assume that the amplitude of the vertical betatron oscillation is smaller compared with the horizontal one. This is practically valid because a high beam injection efficiency and a large momentum acceptance require a wide acceptance mainly in the horizontal plane. We then neglect the terms $O\left(J_{x}^{3}\right)$ and $O\left(J_{y}^{2}\right)$, where $J_{x}$ and $J_{y}$ are the action variables for the horizontal and vertical betatron oscillations. This assumption greatly reduces the number of terms that we have to treat and allows explicit expressions of ADTS. In the following sections, we show the step-by-step details for deriving fourth-order formulas.

\section{B. Second-order perturbation}

As in the first-order treatment, the second-order generating function $G^{(2)}$ is obtained by solving Eq. (25) with $n=2$ :

$$
\begin{gathered}
\left\{\frac{1}{\beta_{x}} \frac{\partial}{\partial \phi_{x}}+\frac{1}{\beta_{y}} \frac{\partial}{\partial \phi_{y}}+\frac{\partial}{\partial s}\right\} G^{(2)}=-V^{(2)} \\
V^{(2)} \equiv \frac{\partial V}{\partial J_{x}} \frac{\partial G^{(1)}}{\partial \phi_{x}}+\frac{\partial V}{\partial J_{y}} \frac{\partial G^{(1)}}{\partial \phi_{y}}-\left\langle\frac{\partial V}{\partial J_{x}} \frac{\partial G^{(1)}}{\partial \phi_{x}}+\frac{\partial V}{\partial J_{y}} \frac{\partial G^{(1)}}{\partial \phi_{y}}\right\rangle
\end{gathered}
$$

and we have used Eq. (50). Every term on the right-hand side of Eq. (67) is known and can be calculated by using Eqs. (31) and (37). The term $V^{(2)}$ is then expressed in the following form:

$V^{(2)}\left(\phi_{x}, J_{x}, \phi_{y}, J_{y}, s\right)=\sum_{m, n=-\infty}^{+\infty} v_{m, n}^{(2)}\left(J_{x}, J_{y}, s\right) e_{m, n}$,

where there are 22 nonzero terms of $v_{m, n}^{(2)}$ and their explicit expressions are given by Eqs. (A1)-(A3) in Appendix A. The second-order generating function is expressed by

$$
G^{(2)}=\sum_{m, n=-\infty}^{+\infty} g_{m, n}^{(2)} e_{m, n},
$$




$$
\begin{aligned}
g_{m, n}^{(2)} \equiv & \frac{i}{2 \sin \pi\left(m \nu_{x}+n \nu_{y}\right)} \\
& \times \int_{s}^{s+C} d s^{\prime} v_{m, n}^{(2)}\left(J_{x}, J_{y}, s^{\prime}\right) e^{i m \Psi_{x}\left(s^{\prime}, s\right)+i n \Psi_{y}\left(s^{\prime}, s\right)}
\end{aligned}
$$

and their expressions are given by Eqs. (A4)-(A6).

The average of the third-order term of the Hamiltonian (23) is calculated as follows:

$$
\begin{aligned}
\left\langle\tilde{H}^{(3)}\right\rangle \equiv & \left\langle\frac{\partial V}{\partial J_{x}} \frac{\partial G^{(2)}}{\partial \phi_{x}}+\frac{\partial V}{\partial J_{y}} \frac{\partial G^{(2)}}{\partial \phi_{y}}+\frac{1}{2} \frac{\partial^{2} V}{\partial J_{x}^{2}}\left(\frac{\partial G^{(1)}}{\partial \phi_{x}}\right)^{2}\right. \\
& \left.+\frac{\partial^{2} V}{\partial J_{x} \partial J_{y}} \frac{\partial G^{(1)}}{\partial \phi_{x}} \frac{\partial G^{(1)}}{\partial \phi_{y}}\right\rangle
\end{aligned}
$$

where we have used the conditions of

$$
\left\langle\frac{\partial G^{(3)}}{\partial \phi_{x}}\right\rangle=\left\langle\frac{\partial G^{(3)}}{\partial \phi_{y}}\right\rangle=0 \quad \text { and } \quad\left\langle\frac{\partial G^{(3)}}{\partial s}\right\rangle=0
$$

By substituting Eqs. (31), (37), and (69) into Eq. (71) we easily find

$$
\left\langle\tilde{H}^{(3)}\right\rangle=0
$$

This means that the third-order term of the Hamiltonian does not contribute to the tune shift, as is well known.

\section{Third-order perturbation}

In a similar manner, the third-order generating function $G^{(3)}$ is obtained by solving

$$
\left\{\frac{1}{\beta_{x}} \frac{\partial}{\partial \phi_{x}}+\frac{1}{\beta_{y}} \frac{\partial}{\partial \phi_{y}}+\frac{\partial}{\partial s}\right\} G^{(3)}=-V^{(3)},
$$

$$
\begin{aligned}
V^{(3)} \equiv & \frac{\partial V}{\partial J_{x}} \frac{\partial G^{(2)}}{\partial \phi_{x}}+\frac{\partial V}{\partial J_{y}} \frac{\partial G^{(2)}}{\partial \phi_{y}}+\frac{1}{2} \frac{\partial^{2} V}{\partial J_{x}^{2}}\left(\frac{\partial G^{(1)}}{\partial \phi_{x}}\right)^{2} \\
& +\frac{\partial^{2} V}{\partial J_{x} \partial J_{y}} \frac{\partial G^{(1)}}{\partial \phi_{x}} \frac{\partial G^{(1)}}{\partial \phi_{y}} \\
\equiv & \sum_{m, n=-\infty}^{+\infty} v_{m, n}^{(3)}\left(J_{x}, J_{y}, s\right) e_{m, n}
\end{aligned}
$$

and we have used Eq. (73). The solution has a form of

$$
\begin{gathered}
G^{(3)}=\sum_{m, n=-\infty}^{+\infty} g_{m, n}^{(3)} e_{m, n}, \\
g_{m, n}^{(3)} \equiv \frac{i}{2 \sin \pi\left(m \nu_{x}+n \nu_{y}\right)} \\
\times \int_{s}^{s+C} d s^{\prime} v_{m, n}^{(3)}\left(J_{x}, J_{y}, s^{\prime}\right) e^{i m \Psi_{x}\left(s^{\prime}, s\right)+i n \Psi_{y}\left(s^{\prime}, s\right)} .
\end{gathered}
$$

There are 46 nonzero terms of $v_{m, n}^{(3)}$, and the expressions of $v_{m, n}^{(3)}$ and $g_{m, n}^{(3)}$ are given in Appendix B.

The fourth-order term of the Hamiltonian is calculated by

$$
\begin{aligned}
\left\langle\tilde{H}^{(4)}\right\rangle= & \left\langle\frac{\partial V}{\partial J_{x}} \frac{\partial G^{(3)}}{\partial \phi_{x}}\right\rangle+\left\langle\frac{\partial V}{\partial J_{y}} \frac{\partial G^{(3)}}{\partial \phi_{y}}\right\rangle+\left\langle\frac{\partial^{2} V}{\partial J_{x}^{2}} \frac{\partial G^{(1)}}{\partial \phi_{x}} \frac{\partial G^{(2)}}{\partial \phi_{x}}\right\rangle+\left\langle\frac{\partial^{2} V}{\partial J_{x} \partial J_{y}} \frac{\partial G^{(1)}}{\partial \phi_{x}} \frac{\partial G^{(2)}}{\partial \phi_{y}}\right\rangle+\left\langle\frac{\partial^{2} V}{\partial J_{x} \partial J_{y}} \frac{\partial G^{(2)}}{\partial \phi_{x}} \frac{\partial G^{(1)}}{\partial \phi_{y}}\right\rangle \\
& +\left\langle\frac{1}{6} \frac{\partial^{3} V}{\partial J_{x}^{3}}\left(\frac{\partial G^{(1)}}{\partial \phi_{x}}\right)^{3}\right\rangle+\left\langle\frac{1}{2} \frac{\partial^{3} V}{\partial J_{x}^{2} \partial J_{y}}\left(\frac{\partial G^{(1)}}{\partial \phi_{x}}\right)^{2} \frac{\partial G^{(1)}}{\partial \phi_{y}}\right\rangle .
\end{aligned}
$$

By substituting Eqs. (31), (37), (69), and (76) into Eq. (78) and collecting $e_{0,0}$-terms, we finally obtain

$$
\begin{aligned}
\left\langle\tilde{H}^{(4)}\right\rangle= & W_{x x x} J_{x}^{3}+W_{x x y} J_{x}^{2} J_{y}+O\left(J_{y}^{2}\right), \\
W_{x x x} \equiv & 2 A_{1} \operatorname{Re}\left[\frac{1}{24}\left(B_{1}^{3}+9 B_{1}^{2} B_{1}^{*}+6 B_{1} B_{1}^{*} B_{2}+9 B_{1}^{2} B_{2}^{*}+18 B_{1} B_{2} B_{2}^{*}+3 B_{2}^{2} B_{2}^{*}\right)\right. \\
& \left.+\left(B_{1}+3 B_{1}^{*}+3 B_{2}^{*}\right) C_{1}+2\left(B_{1}^{*}+3 B_{2}^{*}\right) C_{2}+3 B_{2}^{*} C_{3}-3 D_{1}-3 D_{2}\right],
\end{aligned}
$$




$$
\begin{aligned}
W_{x x y} \equiv & 2 A_{1} \operatorname{Re}\left[-\frac{1}{4}\left(B_{1}^{2} B_{3}+3 B_{1}^{2} B_{3}^{*}+6 B_{1} B_{1}^{*} B_{3}+2 B_{1} B_{2}^{*} B_{3}^{*}+2 B_{1} B_{2} B_{3}^{*}+6 B_{1} B_{2}^{*} B_{3}+6 B_{2} B_{2}^{*} B_{3}\right)\right. \\
& \left.-2\left(B_{3}+3 B_{3}^{*}\right) C_{1}-4 B_{3}^{*} C_{2}+\left(B_{1}+3 B_{1}^{*}+3 B_{2}^{*}\right) C_{4}+2\left(B_{1}^{*}+3 B_{2}^{*}\right) C_{5}-3\left(D_{6}+D_{7}\right)\right] \\
& +2 A_{2} \operatorname{Re}\left\{\frac{3}{4}\left(B_{1}^{2} B_{1}^{*}+B_{1}^{2} B_{2}^{*}+2 B_{1} B_{2} B_{2}^{*}\right)+\frac{1}{2}\left(2 B_{1} B_{1}^{*} B_{4}-2 B_{1} B_{1}^{*} B_{5}+2 B_{1} B_{2}^{*} B_{4}\right.\right. \\
& \left.-2 B_{1} B_{2}^{*} B_{5}+B_{1}^{2} B_{4}^{*}-B_{1}^{2} B_{5}^{*}+2 B_{2} B_{2}^{*} B_{4}-2 B_{2} B_{2}^{*} B_{5}\right)+2\left(B_{1}^{*}+B_{2}^{*}+2 B_{4}^{*}-2 B_{5}^{*}\right) C_{1}+4 B_{2}^{*} C_{2} \\
& \left.+2\left[-\left(B_{1}+B_{1}^{*}\right) C_{6}-\left(B_{1}^{*}+B_{2}^{*}\right) C_{8}+\left(B_{1}^{*}+B_{2}^{*}\right) C_{9}-B_{2}^{*} C_{12}+B_{2}^{*} C_{13}\right]+2\left(D_{1}+2 D_{8}-2 D_{9}\right)\right\},
\end{aligned}
$$

where $C_{i}(s)$ and $D_{i}(s)$ are defined by (B4) and (B8) in Appendix B, respectively.

\section{Fourth-order formulas of ADTS}

Up to the fourth-order in sextupole perturbation, the Hamiltonian is now written as

$$
\tilde{H}=\tilde{H}^{(0)}+\left\langle\tilde{H}^{(2)}\right\rangle+\left\langle\tilde{H}^{(4)}\right\rangle
$$

where $\tilde{H}^{(0)},\left\langle\tilde{H}^{(2)}\right\rangle$, and $\left\langle\tilde{H}^{(4)}\right\rangle$ are given by Eqs. (20), (51) and (79), respectively. We then have the following expressions of higher-order ADTS:

$$
\begin{aligned}
& \tilde{\nu}_{x}=\nu_{x}+2 c_{x x} J_{x}+c_{x y} J_{y}+3 c_{x x x} J_{x}^{2}+2 c_{x x y} J_{x} J_{y}, \\
& \tilde{\nu}_{y}=\nu_{y}+c_{x y} J_{x}+2 c_{y y} J_{y}+c_{x x y} J_{x}^{2}, \\
& c_{\alpha \beta} \equiv \frac{1}{2 \pi} \int_{0}^{C} d s W_{\alpha \beta}(s), \\
& c_{\alpha \beta \gamma} \equiv \frac{1}{2 \pi} \int_{0}^{C} d s W_{\alpha \beta \gamma}(s),
\end{aligned}
$$

where the subscripts $\alpha, \beta$, and $\gamma$ denote $x$ or $y$, and $c_{\alpha \beta}$ and $c_{\alpha \beta \gamma}$ are the second- and fourth-order contributions, respectively. In Eq. (83) the fourth-order term proportional to $J_{y}^{2}$ is neglected, and in Eq. (84) the fourth-order terms proportional to $J_{x} J_{y}$ and $J_{y}^{2}$ are neglected. As explained previously, our main concern is with the behavior of electrons on a median plane where $y=0$, and at $J_{y}=0$ we have

$$
\begin{aligned}
& \tilde{\nu}_{x}=\nu_{x}+2 c_{x x} J_{x}+3 c_{x x x} J_{x}^{2}, \\
& \tilde{\nu}_{y}=\nu_{y}+c_{x y} J_{x}+c_{x x y} J_{x}^{2} .
\end{aligned}
$$

Relations among the horizontal coordinates $\left(x, p_{x}\right)$, $\left(\phi_{x}, I_{x}\right)$, and $\left(\varphi_{x}, J_{x}\right)$ are given by Eqs. (6), (7), (14), and (15). By using Eqs. (13), (69), (70), (A4), (76), (77), and (B5), we have the following expressions for canonical transformation at $J_{y}=0$ :

$$
\begin{aligned}
I_{x}= & J_{x}-2 J_{x}^{3 / 2} \operatorname{Re}\left(B_{1} e_{1,0}+B_{2} e_{3,0}\right) \\
& -4 J_{x}^{2} \operatorname{Re}\left(C_{1} e_{2,0}+2 C_{2} e_{4,0}+3 C_{3} e_{6,0}\right) \\
& -2 J_{x}^{5 / 2} \operatorname{Re}\left(D_{1} e_{1,0}+3 D_{2} e_{3,0}+5 D_{3} e_{5,0}\right. \\
& \left.+7 D_{4} e_{7,0}+9 D_{5} e_{9,0}\right) \\
\varphi_{x}= & \phi_{x}-J_{x}^{1 / 2} \operatorname{Im}\left(3 B_{1} e_{1,0}+B_{2} e_{3,0}\right) \\
& -4 J_{x} \operatorname{Im}\left(C_{1} e_{2,0}+C_{2} e_{4,0}+C_{3} e_{6,0}\right) \\
& -5 J_{x}^{3 / 2} \operatorname{Im}\left(D_{1} e_{1,0}+D_{2} e_{3,0}+D_{3} e_{5,0}\right. \\
& \left.+D_{4} e_{7,0}+D_{5} e_{9,0}\right) .
\end{aligned}
$$

\section{E. Sextupole-separated form of coefficients}

In Eqs. (85) and (86), the sextupole strengths can be separated by rewriting Eqs. (32) and (33) as

$$
\begin{aligned}
A_{1}(s) & =\sum_{i} \lambda_{i} \tilde{A}_{1}^{(i)}(s), \\
A_{2}(s) & =\sum_{i} \lambda_{i} \tilde{A}_{2}^{(i)}(s), \\
\tilde{A}_{1}^{(i)}(s) & \equiv \frac{1}{8 \sqrt{2}} \Delta_{i}(s) \beta_{x}^{3 / 2}(s), \\
\tilde{A}_{2}^{(i)}(s) & \equiv \frac{1}{8 \sqrt{2}} \Delta_{i}(s) \beta_{x}^{1 / 2}(s) \beta_{y}(s),
\end{aligned}
$$

where we have introduced the following step function:

$$
\Delta_{i}(s)= \begin{cases}1, & s_{i} \leq s \leq s_{i}+L_{i} \\ 0, & \text { otherwise }\end{cases}
$$

and $s_{i}, L_{i}$ and $\lambda_{i}=B^{\prime \prime}{ }_{i} /[B \rho]$ are the entrance position, length, and strength of the $i$ th sextupole magnet, respectively. By substituting Eqs. (91) and (92) into the expressions of $B_{i}(s), C_{i}(s)$, and $D_{i}(s)$, we have the following forms for coefficients: 


$$
\begin{aligned}
c_{\alpha \beta} & =\sum_{i, j} \lambda_{i} \lambda_{j} F_{\alpha \beta}^{(i j)}, \\
c_{x x x} & =\sum_{i, j, k, l} \lambda_{i} \lambda_{j} \lambda_{k} \lambda_{l} F_{x x x}^{(i j k l)}, \\
c_{x x y} & =\sum_{i, j, k, l} \lambda_{i} \lambda_{j} \lambda_{k} \lambda_{l} F_{x x y}^{(i j k l)},
\end{aligned}
$$

where $F_{\alpha \beta}^{(i j)}, F_{x x x}^{(i j k l)}$, and $F_{x x y}^{(i j k l)}$ are determined by the Twiss parameters and the betatron phases at sextupole magnet positions and their explicit expressions are given in Appendix C. These types of formulas are useful for sextupole optimization procedures since the evaluation of $F_{\alpha \beta}^{(i j)}, F_{x x x}^{(i j k l)}$, and $F_{x x y}^{(i j k l)}$ is needed only once at the beginning. After this, the coefficients $c_{\alpha \beta}, c_{x x x}$, and $c_{x x y}$ can be calculated immediately for an arbitrary set of $\left\{\lambda_{i}\right\}$.

\section{NUMERICAL EXAMPLES}

As pointed out in the Introduction, the higher-order terms contribute significantly to the motion of circulating electrons when the sextupoles are much stronger than those used in typical third-generation light source storage rings. In this section, we apply our formulas (87) and (88) to a five-bend achromat (5BA) lattice for the SPring- 8 upgrade
[4], where target emittance is about 100 pmrad at $6 \mathrm{GeV}$ (with damping by insertion devices) and the maximum strength of the sextupole magnets is about 6 times larger than that for the current ring. Below, we compare the actual results with the tracking simulations and discuss the effectiveness of the formulas.

In numerical calculations, we divided each sextupole magnet $(0.18 \mathrm{~m}$ or $0.30 \mathrm{~m}$ long) into segments and varied the number of division $N_{\text {div }}$ to check for convergence. For precise calculations of coefficients with at least $0.1 \%$ accuracy, we set $N_{\mathrm{div}}=50$, and for rough estimations with $10 \%-20 \%$ accuracy, we set $N_{\text {div }}=10-20$ in order to save CPU time. We also note that if the ring has a periodic structure with the number of periods $N_{p}$, each coefficient of ADTS is $N_{p}$ times greater than that for a unit structure, and this fact also saves CPU time.

In Fig. 1, we present examples of ADTS for three different sets of sextupole strengths. In these calculations the same linear optics having a bare emittance of 169 pmrad is used, and the ring is composed of 42 unit cells, though the actual ring has four long-straight cells and two injection cells that were neglected for the present purpose of demonstrating the validity of the formulas. In these figures, the fractional part of the horizontal and vertical betatron tunes, $\nu_{x}$ and $\nu_{y}$, are plotted as a function of the normalized horizontal beam position, $x / \sqrt{\beta_{x}}$, where $\beta_{x}(=5.5 \mathrm{~m})$ is the betatron function at an observation point (at the center of a
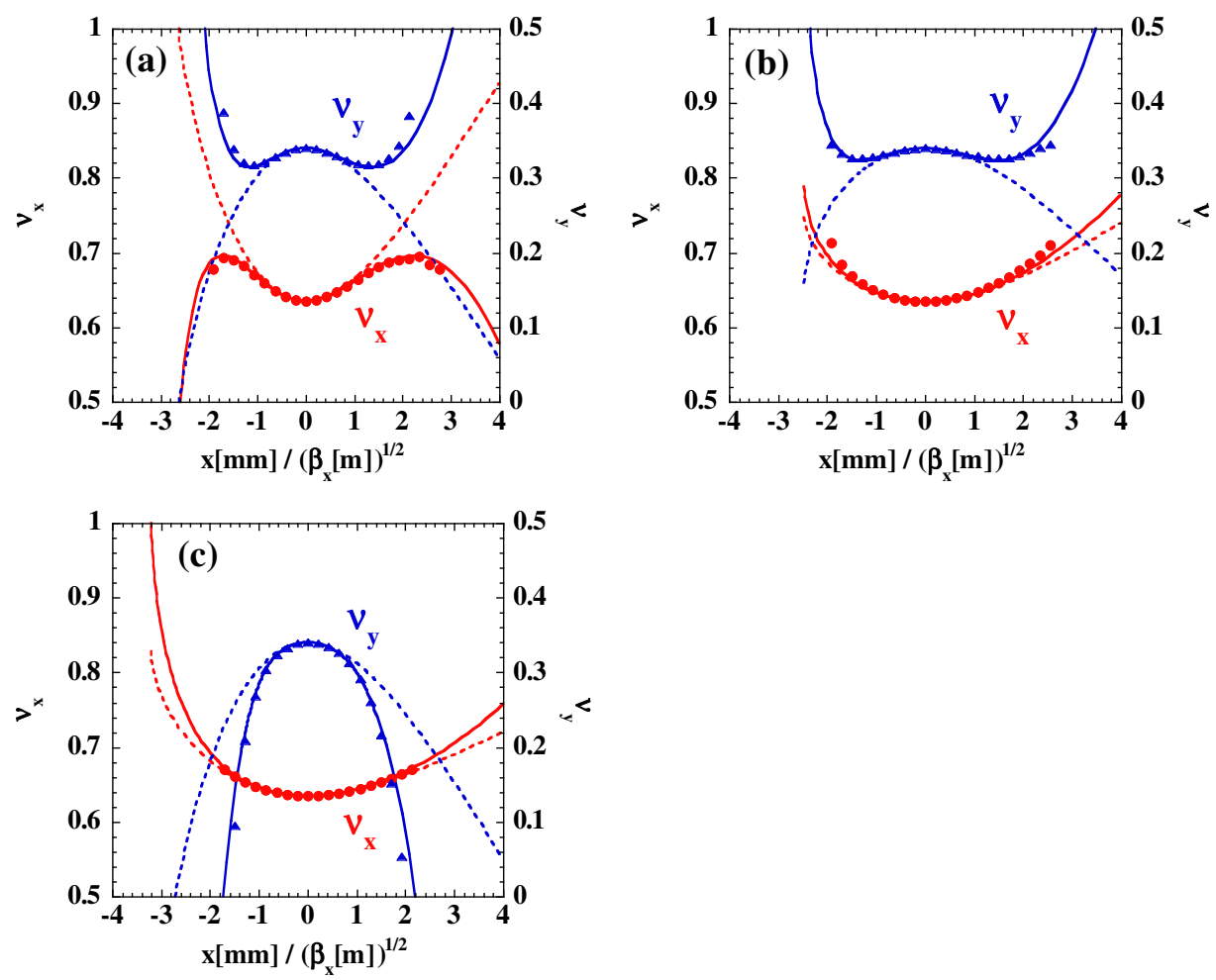

FIG. 1. Examples of ADTS for three different sets of sextupole strengths for the 5BA lattice for the SPring- 8 upgrade. Betatron tunes calculated by the second-order (dashed curves) and fourth-order (solid curves) perturbation formulae are shown together with those by tracking simulations (markers). 


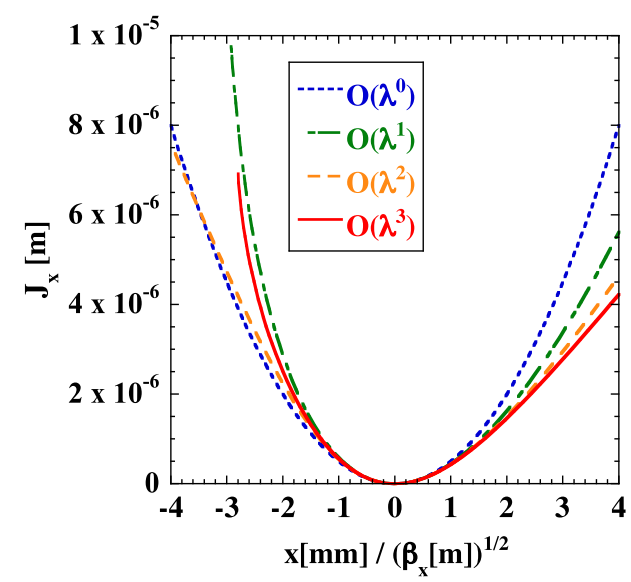

FIG. 2. The relationship between the action variable $J_{x}$ and the normalized coordinate $x / \sqrt{\beta_{x}}$ for the case (a) of Fig. 1 .

straight section). The solid curves show calculations by the perturbation formulas up to $O\left(\lambda^{4}\right)$, dashed curves are for the lowest-order formulas up to $O\left(\lambda^{2}\right)$, and markers show the results of the tracking simulations. We see that the fourth-order formulas explain well the nonlinear behavior of ADTS and that the lowest-order formulas are no longer applicable at large horizontal amplitudes.

The abscissa of Fig. 1 is the normalized coordinate $x / \sqrt{\beta_{x}}$ and it is related to the action variable $J_{x}$ through Eqs. (6) and (89). Figure 2 shows an example of this relationship between $J_{x}$ and $x / \sqrt{\beta_{x}}$ for the case (a) of Fig. 1. In Eq. (89), the coefficients $B_{i}, C_{i}$, and $D_{i}$ are the quantities of $O\left(\lambda^{1}\right), O\left(\lambda^{2}\right)$, and $O\left(\lambda^{3}\right)$, respectively, and each curve in Fig. 2 indicated as $O\left(\lambda^{n}\right)$ corresponds to the truncation of Eq. (89) at the $n$ th-order in sextupole strength. We see that as we proceed to higher orders, the parabolic relation at the lowest order is deformed, which causes the asymmetry of calculated tunes shown in Fig. 1 by the solid and dashed curves.

By using Eqs. (89) and (90), one can also estimate how the horizontal phase-space ellipse is deformed. The results are shown in Fig. 3 by solid curves for the case (a) of Fig. 1. For comparison, we show the results of tracking simulations using red dots. As seen from Eq. (89), the lowest-order correction up to $O\left(\lambda^{1}\right)$ is due to the terms $B_{i}$, introducing a modulation of the ellipse in the form of
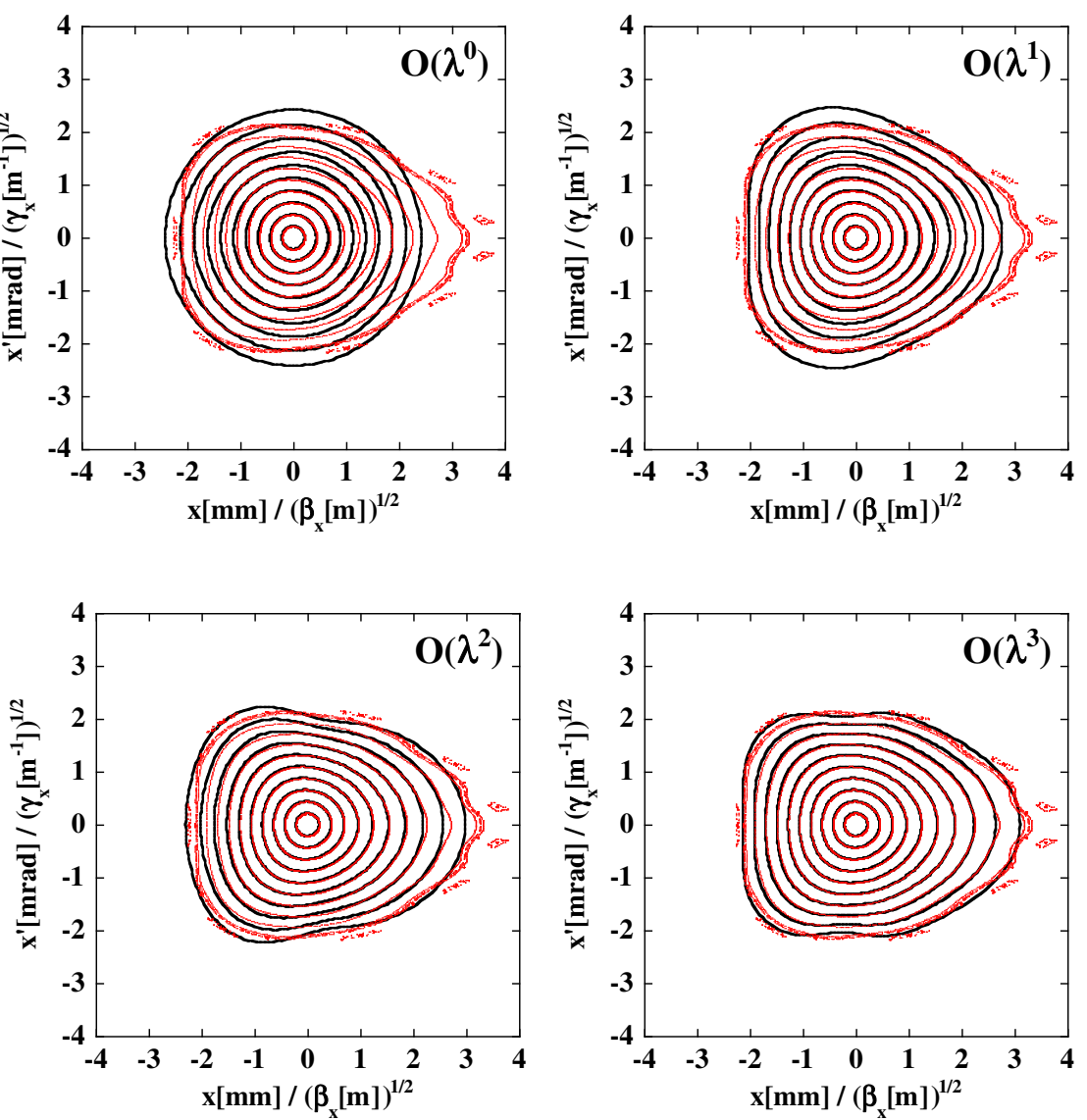

FIG. 3. The horizontal phase-space (Poincare map) for the case (a) of Fig. 1. The position and angle of electrons are normalized by the Twiss parameters $\beta_{x}$ and $\gamma_{x}$ at an observation point. Red dots represent tracking simulation results and solid curves represent perturbation calculations up to the $n$ th-order in sextupole strength, with $n=0,1,2$, and 3 . 
$\exp \left(i \phi_{x}\right)$ and $\exp \left(i 3 \phi_{x}\right)$. As the order of perturbation increases, agreement with the tracking data improves, and at the third-order correction, the phase ellipse modulation in the form up to $\exp \left(i 9 \phi_{x}\right)$ is incorporated and the overall agreement is also satisfactory. The perturbation calculations up to $O\left(\lambda^{3}\right)$ explain the tracking data quite well.

We also checked the applicability of Eqs. (96) to (98) by applying them to a unit cell with eight sextupole magnets. We divided each sextupole magnet into 50 pieces to carry out numerical integrals and evaluated $F_{\alpha \beta}^{(i j)}, F_{x x x}^{(i j k l)}$, and $F_{x x y}^{(i j k l)}$. Though there are $8^{4}$ coefficients for $F_{x x x}^{(i j k l)}$ and $F_{x x y}^{(i j k l)}$, the CPU time required to calculate all of these coefficients was a few tens of seconds when using a standard computer. Once these coefficients are obtained, the objective function in optimization can be evaluated very quickly without doing particle tracking. The storage ring lattice is generally designed to have periodicity and our formulas (96) to (98) are practically applicable to a unit cell structure having a moderate number of sextupole magnets.

\section{SUMMARY}

We developed the explicit expressions of higher-order formulas of ADTS using the canonical perturbation theory up to the fourth-order in sextupole strength, assuming that the amplitude of vertical betatron oscillation is smaller compared with the horizontal one. By applying these formulas to the SPring-8-II lattice having a target emittance of around 100 pmrad, we confirmed that the formulas reproduce well the tracking simulations and that the fourthorder terms in sextupole strength govern the behavior of betatron tunes at large horizontal amplitudes. These results show that the formulas are suitable for understanding and controlling the nonlinear behavior of circulating electrons in a ring with strong sextupole magnets.

\section{ACKNOWLEDGMENTS}

The authors would like to thank M. Takao for useful discussions about canonical perturbation theory.

\section{APPENDIX A: THE SECOND-ORDER GENERATING FUNCTION}

As shown in Eq. (66), $V^{(2)}$ is a "source" for the secondorder generating function $G^{(2)}$. By substituting Eqs. (31) and (37) into Eq. (67) and neglecting the terms of $O\left(J_{y}^{2}\right)$, we obtain $v_{m, n}^{(2)}$ as the following form:

$$
v_{m, n}^{(2)}\left(J_{x}, J_{y}, s\right)=S_{m, n}^{(2)}(s) J_{x}^{2}+T_{m, n}^{(2)}(s) J_{x} J_{y} .
$$

The coefficients $S_{m, n}^{(2)}$ are given by

$$
\begin{aligned}
S_{6,0}^{(2)} & =S_{-6,0}^{(2) *}=-A_{1} B_{2}, \\
S_{4,0}^{(2)} & =S_{-4,0}^{(2) *}=-A_{1}\left(B_{1}+3 B_{2}\right), \\
S_{2,0}^{(2)} & =S_{-2,0}^{(2) *}=-A_{1}\left(3 B_{1}+B_{1}^{*}+3 B_{2}\right), \\
S_{m, n}^{(2)} & =0 \quad \text { for other }(m, n)
\end{aligned}
$$

and $T_{m, n}^{(2)}$ by

$$
\begin{aligned}
T_{4,0}^{(2)}= & T_{-4,0}^{(2) *}=2 A_{1} B_{3}+2 A_{2} B_{2}, \\
T_{2,0}^{(2)}= & T_{-2,0}^{(2) *}=2 A_{1}\left(3 B_{3}+B_{3}^{*}\right) \\
& +2 A_{2}\left(B_{1}+B_{2}-2 B_{4}+2 B_{5}\right), \\
T_{4,2}^{(2)}= & T_{-4,-2}^{(2) *}=A_{1} B_{4}+A_{2} B_{2}, \\
T_{2,2}^{(2)}= & T_{-2,-2}^{(2) *}=A_{1}\left(3 B_{4}+B_{5}^{*}\right)+A_{2}\left(B_{1}+B_{2}-8 B_{4}\right), \\
T_{0,2}^{(2)}= & T_{0,-2}^{(2) *}=3 A_{1}\left(B_{4}+B_{5}^{*}\right) \\
& +A_{2}\left(B_{1}+B_{1}^{*}-8 B_{4}+8 B_{5}^{*}\right), \\
T_{-2,2}^{(2)}= & T_{2,-2}^{(2) *}=A_{1}\left(B_{4}+3 B_{5}^{*}\right)+A_{2}\left(B_{1}^{*}+B_{2}^{*}+8 B_{5}^{*}\right), \\
T_{-4,2}^{(2)}= & T_{4,-2}^{(2) *}=A_{1} B_{5}^{*}+A_{2} B_{2}^{*}, \\
T_{2,4}^{(2)}= & T_{-2,-4}^{(2) *}=-4 A_{2} B_{4}, \\
T_{0,4}^{(2)}= & T_{0,-4}^{(2) *}=-4 A_{2}\left(B_{4}-B_{5}^{*}\right), \\
T_{-2,4}^{(2)}= & T_{2,-4}^{(2) *}=4 A_{2} B_{5}^{*}, \\
T_{m, n}^{(2)}= & 0 \quad \text { for other }(m, n) .
\end{aligned}
$$

The parameters $A_{i}(s)$ and $B_{i}(s)$ are given by Eqs. (32)-(33) and Eqs. (43)-(47), respectively. The second-order generating function $G^{(2)}$ is given by Eqs. (69) and (70), and the function $g_{m, n}^{(2)}$ can be written in the form of

$$
\begin{aligned}
g_{m, n}^{(2)}\left(J_{x}, J_{y}, s\right) & =\sigma_{m, n}^{(2)}(s) J_{x}^{2}+\tau_{m, n}^{(2)}(s) J_{x} J_{y}, \\
\sigma_{m, n}^{(2)}(s)= & \frac{i}{2 \sin \pi\left(m \nu_{x}+n \nu_{y}\right)} \\
& \times \int_{s}^{s+C} d s^{\prime} S_{m, n}^{(2)}\left(s^{\prime}\right) e^{i m \Psi_{x}\left(s^{\prime}, s\right)+i n \Psi_{y}\left(s^{\prime}, s\right)}, \\
\tau_{m, n}^{(2)}(s)= & \frac{i}{2 \sin \pi\left(m \nu_{x}+n \nu_{y}\right)} \\
& \times \int_{s}^{s+C} d s^{\prime} T_{m, n}^{(2)}\left(s^{\prime}\right) e^{i m \Psi_{x}\left(s^{\prime}, s\right)+i n \Psi_{y}\left(s^{\prime}, s\right)} .
\end{aligned}
$$




\section{APPENDIX B: THE THIRD-ORDER GENERATING FUNCTION}

By substituting Eqs. (31), (37) and (69) into Eq. (75) and neglecting the terms of $O\left(J_{y}^{2}\right)$, we obtain the following expression of $v_{m, n}^{(3)}$ :

$$
v_{m, n}^{(3)}\left(J_{x}, J_{y}, s\right)=S_{m, n}^{(3)}(s) J_{x}^{5 / 2}+T_{m, n}^{(3)}(s) J_{x}^{3 / 2} J_{y}
$$

The coefficients $S_{m, n}^{(3)}$ are given by

$$
\begin{aligned}
& S_{9,0}^{(3)}=S_{-9,0}^{(3) *}=A_{1}\left\{\frac{1}{4} B_{2}^{2}-6 C_{3}\right\}, \\
& S_{7,0}^{(3)}=S_{-7,0}^{(3) *}=A_{1}\left\{\frac{1}{4}\left(2 B_{1} B_{2}+3 B_{2}^{2}\right)-4 C_{2}-18 C_{3}\right\}, \\
& S_{5,0}^{(3)}=S_{-5,0}^{(3) *}=A_{1}\left\{\frac{1}{4}\left(B_{1}^{2}+6 B_{1} B_{2}+2 B_{1}^{*} B_{2}+3 B_{2}^{2}\right)-2 C_{1}-12 C_{2}-18 C_{3}\right\}, \\
& S_{3,0}^{(3)}=S_{-3,0}^{(3) *}=A_{1}\left\{\frac{1}{4}\left(3 B_{1}^{2}+2 B_{1} B_{1}^{*}+6 B_{1} B_{2}+6 B_{1}^{*} B_{2}+B_{2}^{2}+2 B_{2} B_{2}^{*}\right)-6 C_{1}-12 C_{2}-6 C_{3}\right\}, \\
& S_{1,0}^{(3)}=S_{-1,0}^{(3) *}=A_{1}\left\{\frac{1}{4}\left(3 B_{1}^{2}+6 B_{1} B_{1}^{*}+B_{1}^{* 2}+2 B_{1} B_{2}+6 B_{1}^{*} B_{2}+2 B_{1} B_{2}^{*}+6 B_{2} B_{2}^{*}\right)-6 C_{1}-2 C_{1}^{*}-4 C_{2}\right\}, \\
& S_{m, n}^{(3)}=0 \text { for other }(m, n)
\end{aligned}
$$

and $T_{m, n}^{(3)}$ by

$$
\begin{aligned}
T_{7,0}^{(3)}= & T_{-7,0}^{(3) *}=-A_{1} B_{2} B_{3}+\frac{1}{2} A_{2} B_{2}^{2}-4 A_{1} C_{5}+12 A_{2} C_{3}, \\
T_{5,0}^{(3)}= & T_{-5,0}^{(3) *}=-A_{1}\left(B_{1} B_{3}+3 B_{2} B_{3}+B_{2} B_{3}^{*}\right)+\frac{1}{2} A_{2}\left(2 B_{1} B_{2}+B_{2}^{2}\right)+2 A_{2}\left(B_{2} B_{4}-B_{2} B_{5}\right) \\
& -2 A_{1}\left(C_{4}+6 C_{5}\right)+4 A_{2}\left(2 C_{2}+3 C_{3}+C_{12}-C_{13}\right), \\
T_{3,0}^{(3)}= & T_{-3,0}^{(3) *}=-A_{1}\left(3 B_{1} B_{3}+B_{1}^{*} B_{3}+3 B_{2} B_{3}+B_{1} B_{3}^{*}+3 B_{2} B_{3}^{*}\right)+\frac{1}{2} A_{2}\left(B_{1}^{2}+2 B_{1} B_{2}+2 B_{1}^{*} B_{2}\right) \\
& +2 A_{2}\left(B_{1} B_{4}+B_{2} B_{4}+B_{2} B_{4}^{*}-B_{1} B_{5}-B_{2} B_{5}-B_{2} B_{5}^{*}\right)-6 A_{1}\left(C_{4}+2 C_{5}\right) \\
& +4 A_{2}\left(C_{1}+2 C_{2}+C_{8}-C_{9}+C_{12}-C_{13}\right), \\
T_{1,0}^{(3)}= & T_{-1,0}^{(3) *}=-A_{1}\left(3 B_{1} B_{3}+3 B_{1}^{*} B_{3}+B_{2} B_{3}+B_{2}^{*} B_{3}+3 B_{1} B_{3}^{*}+B_{1}^{*} B_{3}^{*}+3 B_{2} B_{3}^{*}\right) \\
& +A_{2}\left\{\frac{1}{2}\left(B_{1}^{2}+2 B_{1} B_{1}^{*}+2 B_{1}^{*} B_{2}+2 B_{2} B_{2}^{*}\right)+2\left(B_{1} B_{4}+B_{1}^{*} B_{4}+B_{1} B_{4}^{*}+B_{2} B_{4}^{*}-B_{1} B_{5}-B_{1}^{*} B_{5}-B_{1} B_{5}^{*}-B_{2} B_{5}^{*}\right)\right\} \\
& -2 A_{1}\left(3 C_{4}+C_{4}^{*}+2 C_{5}\right)+4 A_{2}\left(C_{1}+C_{6}+C_{6}^{*}+C_{8}-C_{9}\right), \\
T_{7,2}^{(3)}= & T_{-7,-2}^{(3) *}=-\frac{1}{2} A_{1} B_{2} B_{4}+\frac{1}{4} A_{2} B_{2}^{2}-4 A_{1} C_{12}+6 A_{2} C_{3}, \\
T_{5,2}^{(3)}= & T_{-5,-2}^{(3) *}=-\frac{1}{2} A_{1}\left(B_{1} B_{4}+3 B_{2} B_{4}+B_{2} B_{5}^{*}\right)+A_{2}\left\{\frac{1}{4}\left(2 B_{1} B_{2}+B_{2}^{2}\right)+4 B_{2} B_{4}\right\} \\
& -2 A_{1}\left(C_{8}+6 C_{12}\right)+2 A_{2}\left(2 C_{2}+3 C_{3}+4 C_{12}\right),
\end{aligned}
$$




$$
\begin{aligned}
& T_{3,2}^{(3)}=T_{-3,-2}^{(3) *}=-\frac{1}{2} A_{1}\left(3 B_{1} B_{4}+B_{1}^{*} B_{4}+3 B_{2} B_{4}+B_{1} B_{5}^{*}+3 B_{2} B_{5}^{*}\right) \\
& +A_{2}\left\{\frac{1}{4}\left(B_{1}^{2}+2 B_{1} B_{2}+2 B_{1}^{*} B_{2}\right)+4\left(B_{1} B_{4}+B_{2} B_{4}-B_{2} B_{5}^{*}\right)\right\} \\
& -6 A_{1}\left(C_{8}+2 C_{12}\right)+2 A_{2}\left(C_{1}+2 C_{2}+4 C_{8}+4 C_{10}+4 C_{12}\right), \\
& T_{1,2}^{(3)}=T_{-1,-2}^{(3) *}=-\frac{1}{2} A_{1}\left(3 B_{1} B_{4}+3 B_{1}^{*} B_{4}+B_{2} B_{4}+B_{2}^{*} B_{4}+3 B_{1} B_{5}^{*}+B_{1}^{*} B_{5}^{*}+3 B_{2} B_{5}^{*}\right) \\
& +A_{2}\left\{\frac{1}{4}\left(B_{1}^{2}+2 B_{1} B_{1}^{*}+2 B_{1}^{*} B_{2}+2 B_{2} B_{2}^{*}\right)+4\left(B_{1} B_{4}+B_{1}^{*} B_{4}-B_{1} B_{5}^{*}-B_{2} B_{5}^{*}\right)\right\} \\
& -2 A_{1}\left(3 C_{8}+C_{9}^{*}+2 C_{12}\right)+2 A_{2}\left(C_{1}+4 C_{6}+4 C_{7}+4 C_{8}+4 C_{10}\right) \text {, } \\
& T_{-1,2}^{(3)}=T_{1,-2}^{(3) *}=-\frac{1}{2} A_{1}\left(B_{1} B_{4}+3 B_{1}^{*} B_{4}+3 B_{2}^{*} B_{4}+3 B_{1} B_{5}^{*}+3 B_{1}^{*} B_{5}^{*}+B_{2} B_{5}^{*}+B_{2}^{*} B_{5}^{*}\right) \\
& +A_{2}\left\{\frac{1}{4}\left(2 B_{1} B_{1}^{*}+B_{1}^{* 2}+2 B_{1} B_{2}^{*}+2 B_{2} B_{2}^{*}\right)+4\left(B_{1}^{*} B_{4}+B_{2}^{*} B_{4}-B_{1} B_{5}^{*}-B_{1}^{*} B_{5}^{*}\right)\right\} \\
& -2 A_{1}\left(C_{8}+3 C_{9}^{*}+2 C_{13}^{*}\right)+2 A_{2}\left(C_{1}^{*}+4 C_{6}+4 C_{7}-4 C_{9}^{*}-4 C_{11}^{*}\right), \\
& T_{-3,2}^{(3)}=T_{3,-2}^{(3) *}=-\frac{1}{2} A_{1}\left(B_{1}^{*} B_{4}+3 B_{2}^{*} B_{4}+B_{1} B_{5}^{*}+3 B_{1}^{*} B_{5}^{*}+3 B_{2}^{*} B_{5}^{*}\right) \\
& +A_{2}\left\{\frac{1}{4}\left(B_{1}^{* 2}+2 B_{1} B_{2}^{*}+2 B_{1}^{*} B_{2}^{*}\right)+4\left(B_{2}^{*} B_{4}-B_{1}^{*} B_{5}^{*}-B_{2}^{*} B_{5}^{*}\right)\right\} \\
& -6 A_{1}\left(C_{9}^{*}+2 C_{13}^{*}\right)+2 A_{2}\left(C_{1}^{*}+2 C_{2}^{*}-4 C_{9}^{*}-4 C_{11}^{*}-4 C_{13}^{*}\right), \\
& T_{-5,2}^{(3)}=T_{5,-2}^{(3) *}=-\frac{1}{2} A_{1}\left(B_{2}^{*} B_{4}+B_{1}^{*} B_{5}^{*}+3 B_{2}^{*} B_{5}^{*}\right)+A_{2}\left\{\frac{1}{4}\left(2 B_{1}^{*} B_{2}^{*}+B_{2}^{* 2}\right)-4 B_{2}^{*} B_{5}^{*}\right\} \\
& -2 A_{1}\left(C_{9}^{*}+6 C_{13}^{*}\right)+2 A_{2}\left(2 C_{2}^{*}+3 C_{3}^{*}-4 C_{13}^{*}\right), \\
& T_{-7,2}^{(3)}=T_{7,-2}^{(3) *}=-\frac{1}{2} A_{1} B_{2}^{*} B_{5}^{*}+\frac{1}{4} A_{2} B_{2}^{* 2}-4 A_{1} C_{13}^{*}+6 A_{2} C_{3}^{*} \text {, } \\
& T_{5,4}^{(3)}=T_{-5,-4}^{(3) *}=2 A_{2} B_{2} B_{4}-2 A_{1} C_{10}+4 A_{2} C_{12}, \\
& T_{3,4}^{(3)}=T_{-3,-4}^{(3) *}=2 A_{2}\left(B_{1} B_{4}+B_{2} B_{4}-B_{2} B_{5}^{*}\right)-6 A_{1} C_{10}+4 A_{2}\left(C_{8}+4 C_{10}+C_{12}\right), \\
& T_{1,4}^{(3)}=T_{-1,-4}^{(3) *}=2 A_{2}\left(B_{1} B_{4}+B_{1}^{*} B_{4}-B_{1} B_{5}^{*}-B_{2} B_{5}^{*}\right)-2 A_{1}\left(3 C_{10}+C_{11}^{*}\right)+4 A_{2}\left(C_{6}+4 C_{7}+C_{8}+4 C_{10}\right), \\
& T_{-1,4}^{(3)}=T_{1,-4}^{(3) *}=2 A_{2}\left(B_{1}^{*} B_{4}+B_{2}^{*} B_{4}-B_{1} B_{5}^{*}-B_{1}^{*} B_{5}^{*}\right)-2 A_{1}\left(C_{10}+3 C_{11}^{*}\right)+4 A_{2}\left(C_{6}+4 C_{7}-C_{9}^{*}-4 C_{11}^{*}\right) \text {, } \\
& T_{-3,4}^{(3)}=T_{3,-4}^{(3) *}=2 A_{2}\left(B_{2}^{*} B_{4}-B_{1}^{*} B_{5}^{*}-B_{2}^{*} B_{5}^{*}\right)-6 A_{1} C_{11}^{*}-4 A_{2}\left(C_{9}^{*}+4 C_{11}^{*}+C_{13}^{*}\right), \\
& T_{-5,4}^{(3)}=T_{5,-4}^{(3) *}=-2 A_{2} B_{2}^{*} B_{5}^{*}-2 A_{1} C_{11}^{*}-4 A_{2} C_{13}^{*} \text {, } \\
& T_{3,6}^{(3)}=T_{-3,-6}^{(3) *}=8 A_{2} C_{10}, \\
& T_{1,6}^{(3)}=T_{-1,-6}^{(3) *}=8 A_{2}\left(C_{7}+C_{10}\right), \\
& T_{-1,6}^{(3)}=T_{1,-6}^{(3) *}=8 A_{2}\left(C_{7}-C_{11}^{*}\right), \\
& T_{-3,6}^{(3)}=T_{3,-6}^{(3) *}=-8 A_{2} C_{11}^{*} \text {, } \\
& T_{m, n}^{(3)}=0 \quad \text { for other }(m, n) \text {. }
\end{aligned}
$$


In the above, we have defined

$$
\begin{array}{rlrl}
C_{1}(s) & \equiv-i \sigma_{2,0}^{(2)}(s), & C_{2}(s) \equiv-i \sigma_{4,0}^{(2)}(s), & C_{3}(s) \equiv-i \sigma_{6,0}^{(2)}(s), \\
C_{4}(s) \equiv-i \tau_{2,0}^{(2)}(s), & C_{5}(s) \equiv-i \tau_{4,0}^{(2)}(s), & C_{6}(s) \equiv-i \tau_{0,2}^{(2)}(s), \quad C_{7}(s) \equiv-i \tau_{0,4}^{(2)}(s), \\
C_{8}(s) \equiv-i \tau_{2,2}^{(2)}(s), & C_{9}(s) \equiv-i \tau_{2,-2}^{(2)}(s), & C_{10}(s) \equiv-i \tau_{2,4}^{(2)}(s), \quad C_{11}(s) \equiv-i \tau_{2,-4}^{(2)}(s), \\
C_{12}(s) \equiv-i \tau_{4,2}^{(2)}(s), & C_{13}(s) \equiv-i \tau_{4,-2}^{(2)}(s) &
\end{array}
$$

and $\sigma_{m, n}^{(2)}(s)$ and $\tau_{m, n}^{(2)}(s)$ are given by (A5) and (A6), respectively. The third-order generating function $G^{(3)}$ is given by Eqs. (76) and (77), and the function $g_{m, n}^{(3)}$ has a form of

$$
\begin{aligned}
g_{m, n}^{(3)}\left(J_{x}, J_{y}, s\right) & =\sigma_{m, n}^{(3)}(s) J_{x}^{5 / 2}+\tau_{m, n}^{(3)}(s) J_{x}^{3 / 2} J_{y}, \\
\sigma_{m, n}^{(3)} & =\frac{i}{2 \sin \pi\left(m \nu_{x}+n \nu_{y}\right)} \int_{s}^{s+C} d s^{\prime} S_{m, n}^{(3)}\left(s^{\prime}\right) e^{i m \Psi_{x}\left(s^{\prime}, s\right)+i n \Psi_{y}\left(s^{\prime}, s\right)}, \\
\tau_{m, n}^{(3)} & =\frac{i}{2 \sin \pi\left(m \nu_{x}+n \nu_{y}\right)} \int_{s}^{s+C} d s^{\prime} T_{m, n}^{(3)}\left(s^{\prime}\right) e^{i m \Psi_{x}\left(s^{\prime}, s\right)+i n \Psi_{y}\left(s^{\prime}, s\right)} .
\end{aligned}
$$

For later use, we define the following:

$$
\begin{aligned}
& D_{1}(s) \equiv-i \sigma_{1,0}^{(3)}(s), \quad D_{2}(s) \equiv-i \sigma_{3,0}^{(3)}(s), \quad D_{3}(s) \equiv-i \sigma_{5,0}^{(3)}(s), \quad D_{4}(s) \equiv-i \sigma_{7,0}^{(3)}(s), \quad D_{5}(s) \equiv-i \sigma_{9,0}^{(3)}(s), \\
& D_{6}(s) \equiv-i \tau_{1,0}^{(3)}(s), \quad D_{7}(s) \equiv-i \tau_{3,0}^{(3)}(s), \quad D_{8}(s) \equiv-i \tau_{1,2}^{(3)}(s), \quad D_{9}(s) \equiv-i \tau_{1,-2}^{(3)}(s) .
\end{aligned}
$$

\section{APPENDIX C: EXPLICIT EXPRESSIONS OF $F_{\alpha \beta}^{(i j)}, F_{x x x}^{(i j k l)}$, AND $F_{x x y}^{(i j k l)}$}

With the use of Eqs. (91) and (92) we have

$$
\begin{aligned}
B_{n}(s) & =\sum_{i} \lambda_{i} \tilde{B}_{n}^{(i)}(s), \\
C_{n}(s) & =\sum_{i, j} \lambda_{i} \lambda_{j} \tilde{C}_{n}^{(i j)}(s), \\
D_{n}(s) & =\sum_{i, j, k} \lambda_{i} \lambda_{j} \lambda_{k} \tilde{D}_{n}^{(i j k)}(s),
\end{aligned}
$$

where

$$
\begin{aligned}
& \tilde{B}_{1}^{(i)}(s) \equiv \frac{1}{\sin \pi \nu_{x}} \int_{s}^{s+C} d s^{\prime} \tilde{A}_{1}^{(i)}\left(s^{\prime}\right) e^{i \Psi_{x}\left(s^{\prime}, s\right)}, \quad \tilde{B}_{2}^{(i)}(s) \equiv \frac{1}{\sin 3 \pi \nu_{x}} \int_{s}^{s+C} d s^{\prime} \tilde{A}_{1}^{(i)}\left(s^{\prime}\right) e^{i 3 \Psi_{x}\left(s^{\prime}, s\right)}, \\
& \tilde{B}_{3}^{(i)}(s) \equiv \frac{1}{\sin \pi \nu_{x}} \int_{s}^{s+C} d s^{\prime} \tilde{A}_{2}^{(i)}\left(s^{\prime}\right) e^{i \Psi_{x}\left(s^{\prime}, s\right)}, \quad \tilde{B}_{4}^{(i)}(s) \equiv \frac{1}{\sin \pi\left(\nu_{x}+2 \nu_{y}\right)} \int_{s}^{s+C} d s^{\prime} \tilde{A}_{2}^{(i)}\left(s^{\prime}\right) e^{i\left\{\Psi_{x}\left(s^{\prime}, s\right)+2 \Psi_{y}\left(s^{\prime}, s\right)\right\}} \\
& \tilde{B}_{5}^{(i)}(s) \equiv \frac{1}{\sin \pi\left(\nu_{x}-2 \nu_{y}\right)} \int_{s}^{s+C} d s^{\prime} \tilde{A}_{2}^{(i)}\left(s^{\prime}\right) e^{i\left\{\Psi_{x}\left(s^{\prime}, s\right)-2 \Psi_{y}\left(s^{\prime}, s\right)\right\}},
\end{aligned}
$$




$$
\begin{aligned}
& \tilde{C}_{1}^{(i j)}(s) \equiv \frac{1}{2 \sin 2 \pi \nu_{x}} \int_{s}^{s+C} d s^{\prime}\left[-\tilde{A}_{1}^{(i)}\left(3 \tilde{B}_{1}^{(j)}+\tilde{B}_{1}^{(j) *}+3 \tilde{B}_{2}^{(j)}\right)\right]_{s^{\prime}} e^{i 2 \Psi_{x}\left(s^{\prime}, s\right)}, \\
& \tilde{C}_{2}^{(i j)}(s) \equiv \frac{1}{2 \sin 4 \pi \nu_{x}} \int_{s}^{s+C} d s^{\prime}\left[-\tilde{A}_{1}^{(i)}\left(\tilde{B}_{1}^{(j)}+3 \tilde{B}_{2}^{(j)}\right)\right]_{s^{\prime}} e^{i 4 \Psi_{x}\left(s^{\prime}, s\right)}, \\
& \tilde{C}_{3}^{(i j)}(s) \equiv \frac{1}{2 \sin 6 \pi \nu_{x}} \int_{s}^{s+C} d s^{\prime}\left[-\tilde{A}_{1}^{(i)} \tilde{B}_{2}^{(j)}\right]_{s^{\prime}} e^{i 6 \Psi_{x}\left(s^{\prime}, s\right)}, \\
& \tilde{C}_{4}^{(i j)}(s) \equiv \frac{1}{2 \sin 2 \pi \nu_{x}} \int_{s}^{s+C} d s^{\prime}\left[2 \tilde{A}_{1}^{(i)}\left(3 \tilde{B}_{3}^{(j)}+\tilde{B}_{3}^{(j) *}\right)+2 \tilde{A}_{2}^{(i)}\left(\tilde{B}_{1}^{(j)}+\tilde{B}_{2}^{(j)}-2 \tilde{B}_{4}^{(j)}+2 \tilde{B}_{5}^{(j)}\right)\right]_{s^{\prime}} e^{i 2 \Psi_{x}\left(s^{\prime}, s\right)}, \\
& \tilde{C}_{5}^{(i j)}(s) \equiv \frac{1}{2 \sin 4 \pi \nu_{x}} \int_{s}^{s+C} d s^{\prime}\left[2 \tilde{A}_{1}^{(i)} \tilde{B}_{3}^{(j)}+2 \tilde{A}_{2}^{(i)} \tilde{B}_{2}^{(j)}\right]_{s^{\prime}} e^{i 4 \Psi_{x}\left(s^{\prime}, s\right)} \\
& \tilde{C}_{6}^{(i j)}(s) \equiv \frac{1}{2 \sin 2 \pi \nu_{y}} \int_{s}^{s+C} d s^{\prime}\left[3 \tilde{A}_{1}^{(i)}\left(\tilde{B}_{4}^{(j)}+\tilde{B}_{5}^{(j) *}\right)+\tilde{A}_{2}^{(i)}\left(\tilde{B}_{1}^{(j)}+\tilde{B}_{1}^{(j) *}-8 \tilde{B}_{4}^{(j)}+8 \tilde{B}_{5}^{(j) *}\right)\right]_{s} e^{i 2 \Psi_{y}\left(s^{\prime}, s\right)}, \\
& \tilde{C}_{7}^{(i j)}(s) \equiv \frac{1}{2 \sin 4 \pi \nu_{y}} \int_{s}^{s+C} d s^{\prime}\left[-4 \tilde{A}_{2}^{(i)}\left(\tilde{B}_{4}^{(j)}-\tilde{B}_{5}^{(j) *}\right)\right]_{s^{\prime}} e^{i 4 \Psi_{y}\left(s^{\prime}, s\right)} \\
& \tilde{C}_{8}^{(i j)}(s) \equiv \frac{1}{2 \sin 2 \pi\left(\nu_{x}+\nu_{y}\right)} \int_{s}^{s+C} d s^{\prime}\left[\tilde{A}_{1}^{(i)}\left(3 \tilde{B}_{4}^{(j)}+\tilde{B}_{5}^{(j) *}\right)+\tilde{A}_{2}^{(i)}\left(\tilde{B}_{1}^{(j)}+\tilde{B}_{2}^{(j)}-8 \tilde{B}_{4}^{(j)}\right)\right]_{s^{\prime}} e^{i 2 \Psi_{x}\left(s^{\prime}, s\right)+i 2 \Psi_{y}\left(s^{\prime}, s\right)}, \\
& \tilde{C}_{9}^{(i j)}(s) \equiv \frac{1}{2 \sin 2 \pi\left(\nu_{x}-\nu_{y}\right)} \int_{s}^{s+C} d s^{\prime}\left[\tilde{A}_{1}^{(i)}\left(\tilde{B}_{4}^{(j) *}+3 \tilde{B}_{5}^{(j)}\right)+\tilde{A}_{2}^{(i)}\left(\tilde{B}_{1}^{(j)}+\tilde{B}_{2}^{(j)}+8 \tilde{B}_{5}^{(j)}\right)\right]_{s^{\prime}} e^{i 2 \Psi_{x}\left(s^{\prime}, s\right)-i 2 \Psi_{y}\left(s^{\prime}, s\right)}, \\
& \tilde{C}_{10}^{(i j)}(s) \equiv \frac{1}{2 \sin 2 \pi\left(\nu_{x}+2 \nu_{y}\right)} \int_{s}^{s+C} d s^{\prime}\left[-4 \tilde{A}_{2}^{(i)} \tilde{B}_{4}^{(j)}\right]_{s^{\prime}} e^{i 2 \Psi_{x}\left(s^{\prime}, s\right)+i 4 \Psi_{y}\left(s^{\prime}, s\right)}, \\
& \tilde{C}_{11}^{(i j)}(s) \equiv \frac{1}{2 \sin 2 \pi\left(\nu_{x}-2 \nu_{y}\right)} \int_{s}^{s+C} d s^{\prime}\left[4 \tilde{A}_{2}^{(i)} \tilde{B}_{5}^{(j)}\right]_{s^{\prime}} e^{i 2 \Psi_{x}\left(s^{\prime}, s\right)-i 4 \Psi_{y}\left(s^{\prime}, s\right)}, \\
& \tilde{C}_{12}^{(i j)}(s) \equiv \frac{1}{2 \sin 2 \pi\left(2 \nu_{x}+\nu_{y}\right)} \int_{s}^{s+C} d s^{\prime}\left[\tilde{A}_{1}^{(i)} \tilde{B}_{4}^{(j)}+\tilde{A}_{2}^{(i)} \tilde{B}_{2}^{(j)}\right]_{s^{\prime}} e^{i 4 \Psi_{x}\left(s^{\prime}, s\right)+i 2 \Psi_{y}\left(s^{\prime}, s\right)}, \\
& \tilde{C}_{13}^{(i j)}(s) \equiv \frac{1}{2 \sin 2 \pi\left(2 \nu_{x}-\nu_{y}\right)} \int_{s}^{s+C} d s^{\prime}\left[\tilde{A}_{1}^{(i)} \tilde{B}_{5}^{(j)}+\tilde{A}_{2}^{(i)} \tilde{B}_{2}^{(j)}\right]_{s^{\prime}} e^{i 4 \Psi_{x}\left(s^{\prime}, s\right)-i 2 \Psi_{y}\left(s^{\prime}, s\right)},
\end{aligned}
$$




$$
\begin{aligned}
& \tilde{D}_{1}^{(i j k)}(s) \equiv \frac{1}{2 \sin \pi \nu_{x}} \int_{s}^{s+C} d s^{\prime}\left[\tilde { A } _ { 1 } ^ { ( i ) } \left\{\frac{1}{4}\left(3 \tilde{B}_{1}^{(j)} \tilde{B}_{1}^{(k)}+6 \tilde{B}_{1}^{(j)} \tilde{B}_{1}^{(k) *}+\tilde{B}_{1}^{(j) *} \tilde{B}_{1}^{(k) *}+2 \tilde{B}_{1}^{(j)} \tilde{B}_{2}^{(k)}+6 \tilde{B}_{1}^{(j) *} \tilde{B}_{2}^{(k)}+2 \tilde{B}_{1}^{(j)} \tilde{B}_{2}^{(k) *}+6 \tilde{B}_{2}^{(j)} \tilde{B}_{2}^{(k) *}\right)\right.\right. \\
& \left.\left.-6 \tilde{C}_{1}^{(j k)}-2 \tilde{C}_{1}^{(j k) *}-4 \tilde{C}_{2}^{(j k)}\right\}\right]_{s^{\prime}} e^{i \Psi_{x}\left(s^{\prime}, s\right)}, \\
& \tilde{D}_{2}^{(i j k)}(s) \equiv \frac{1}{2 \sin 3 \pi \nu_{x}} \int_{s}^{s+C} d s^{\prime}\left[\tilde { A } _ { 1 } ^ { ( i ) } \left\{\frac{1}{4}\left(3 \tilde{B}_{1}^{(j)} \tilde{B}_{1}^{(k)}+2 \tilde{B}_{1}^{(j)} \tilde{B}_{1}^{(k) *}+6 \tilde{B}_{1}^{(j)} \tilde{B}_{2}^{(k)}+6 \tilde{B}_{1}^{(j) *} \tilde{B}_{2}^{(k)}+\tilde{B}_{2}^{(j)} \tilde{B}_{2}^{(k)}+2 \tilde{B}_{2}^{(j)} \tilde{B}_{2}^{(k) *}\right)\right.\right. \\
& \left.\left.-6 \tilde{C}_{1}^{(j k)}-12 \tilde{C}_{2}^{(j k)}-6 \tilde{C}_{3}^{(j k)}\right\}\right]_{s^{\prime}} e^{i 3 \Psi_{x}\left(s^{\prime}, s\right)}, \\
& \tilde{D}_{3}^{(i j k)}(s) \equiv \frac{1}{2 \sin 5 \pi \nu_{x}} \int_{s}^{s+C} d s^{\prime}\left[\tilde{A}_{1}^{(i)}\left\{\frac{1}{4}\left(\tilde{B}_{1}^{(j)} \tilde{B}_{1}^{(k)}+6 \tilde{B}_{1}^{(j)} \tilde{B}_{2}^{(k)}+2 \tilde{B}_{1}^{(j) *} \tilde{B}_{2}^{(k)}+3 \tilde{B}_{2}^{(j)} \tilde{B}_{2}^{(k)}\right)-2 \tilde{C}_{1}^{(j k)}-12 \tilde{C}_{2}^{(j k)}-18 \tilde{C}_{3}^{(j k)}\right\}\right]_{s^{\prime}} e^{i 5 \Psi_{x}\left(s^{\prime}, s\right)} \\
& \tilde{D}_{4}^{(i j k)}(s) \equiv \frac{1}{2 \sin 7 \pi \nu_{x}} \int_{s}^{s+C} d s^{\prime}\left[\tilde{A}_{1}^{(i)}\left\{\frac{1}{4}\left(2 \tilde{B}_{1}^{(j)} \tilde{B}_{2}^{(k)}+3 \tilde{B}_{2}^{(j)} \tilde{B}_{2}^{(k)}\right)-4 \tilde{C}_{2}^{(j k)}-18 \tilde{C}_{3}^{(j k)}\right\}\right]_{s^{\prime}} e^{i 7 \Psi_{x}\left(s^{\prime}, s\right)}, \\
& \tilde{D}_{5}^{(i j k)}(s) \equiv \frac{1}{2 \sin 9 \pi \nu_{x}} \int_{s}^{s+C} d s^{\prime}\left[\tilde{A}_{1}^{(i)}\left\{\frac{1}{4} \tilde{B}_{2}^{(j)} \tilde{B}_{2}^{(k)}-6 \tilde{C}_{3}^{(j k)}\right\}\right]_{s^{\prime}} e^{i \vartheta \Psi_{x}\left(s^{\prime}, s\right)} \\
& \tilde{D}_{6}^{(i j k)}(s) \equiv \frac{1}{2 \sin \pi \nu_{x}} \int_{s}^{s+C} d s^{\prime}\left[-\tilde{A}_{1}^{(i)}\left(3 \tilde{B}_{1}^{(j)} \tilde{B}_{3}^{(k)}+3 \tilde{B}_{1}^{(j) *} \tilde{B}_{3}^{(k)}+\tilde{B}_{2}^{(j)} \tilde{B}_{3}^{(k)}+\tilde{B}_{2}^{(j) *} \tilde{B}_{3}^{(k)}+3 \tilde{B}_{1}^{(j)} \tilde{B}_{3}^{(k) *}+\tilde{B}_{1}^{(j) *} \tilde{B}_{3}^{(k) *}+3 \tilde{B}_{2}^{(j)} \tilde{B}_{3}^{(k) *}\right)\right. \\
& +\tilde{A}_{2}^{(i)}\left\{\frac{1}{2}\left(\tilde{B}_{1}^{(j)} \tilde{B}_{1}^{(k)}+2 \tilde{B}_{1}^{(j)} \tilde{B}_{1}^{(k) *}+2 \tilde{B}_{1}^{(j) *} \tilde{B}_{2}^{(k)}+2 \tilde{B}_{2}^{(j)} \tilde{B}_{2}^{(k) *}\right)\right. \\
& \left.+2\left(\tilde{B}_{1}^{(j)} \tilde{B}_{4}^{(k)}+\tilde{B}_{1}^{(j) *} \tilde{B}_{4}^{(k)}+\tilde{B}_{1}^{(j)} \tilde{B}_{4}^{(k) *}+\tilde{B}_{2}^{(j)} \tilde{B}_{4}^{(k) *}-\tilde{B}_{1}^{(j)} \tilde{B}_{5}^{(k)}-\tilde{B}_{1}^{(j) *} \tilde{B}_{5}^{(k)}-\tilde{B}_{1}^{(j)} \tilde{B}_{5}^{(k) *}-\tilde{B}_{2}^{(j)} \tilde{B}_{5}^{(k) *}\right)\right\} \\
& \left.-2 \tilde{A}_{1}^{(i)}\left(3 \tilde{C}_{4}^{(j k)}+\tilde{C}_{4}^{(j k) *}+2 \tilde{C}_{5}^{(j k)}\right)+4 \tilde{A}_{2}^{(i)}\left(\tilde{C}_{1}^{(j k)}+\tilde{C}_{6}^{(j k)}+\tilde{C}_{6}^{(j k) *}+\tilde{C}_{8}^{(j k)}-\tilde{C}_{9}^{(j k)}\right)\right]_{s^{\prime}} e^{i \Psi_{x}\left(s^{\prime}, s\right)}, \\
& \tilde{D}_{7}^{(i j k)}(s) \equiv \frac{1}{2 \sin 3 \pi \nu_{x}} \int_{s}^{s+C} d s^{\prime} \times\left[-\tilde{A}_{1}^{(i)}\left(3 \tilde{B}_{1}^{(j)} \tilde{B}_{3}^{(k)}+\tilde{B}_{1}^{(j) *} \tilde{B}_{3}^{(k)}+3 \tilde{B}_{2}^{(j)} \tilde{B}_{3}^{(k)}+\tilde{B}_{1}^{(j)} \tilde{B}_{3}^{(k) *}+3 \tilde{B}_{2}^{(j)} \tilde{B}_{3}^{(k) *}\right)\right. \\
& +\frac{1}{2} \tilde{A}_{2}^{(i)}\left(\tilde{B}_{1}^{(j)} \tilde{B}_{1}^{(k)}+2 \tilde{B}_{1}^{(j)} \tilde{B}_{2}^{(k)}+2 \tilde{B}_{1}^{(j) *} \tilde{B}_{2}^{(k)}\right)+2 \tilde{A}_{2}^{(i)}\left(\tilde{B}_{1}^{(j)} \tilde{B}_{4}^{(k)}+\tilde{B}_{2}^{(j)} \tilde{B}_{4}^{(k)}+\tilde{B}_{2}^{(j)} \tilde{B}_{4}^{(k) *}-\tilde{B}_{1}^{(j)} \tilde{B}_{5}^{(k)}-\tilde{B}_{2}^{(j)} \tilde{B}_{5}^{(k)}-\tilde{B}_{2}^{(j)} \tilde{B}_{5}^{(k) *}\right) \\
& \left.-6 \tilde{A}_{1}^{(i)}\left(\tilde{C}_{4}^{(j k)}+2 \tilde{C}_{5}^{(j k)}\right)+4 \tilde{A}_{2}^{(i)}\left(\tilde{C}_{1}^{(j k)}+2 \tilde{C}_{2}^{(j k)}+\tilde{C}_{8}^{(j k)}-\tilde{C}_{9}^{(j k)}+\tilde{C}_{12}^{(j k)}-\tilde{C}_{13}^{(j k)}\right)\right]_{s^{\prime}} e^{i 3 \Psi_{x}\left(s^{\prime}, s\right)}, \\
& \tilde{D}_{8}^{(i j k)}(s) \equiv \frac{1}{2 \sin \pi\left(\nu_{x}+2 \nu_{y}\right)} \int_{s}^{s+C} d s^{\prime} \times\left[-\frac{1}{2} \tilde{A}_{1}^{(i)}\left(3 \tilde{B}_{1}^{(j)} \tilde{B}_{4}^{(k)}+3 \tilde{B}_{1}^{(j) *} \tilde{B}_{4}^{(k)}+\tilde{B}_{2}^{(j)} \tilde{B}_{4}^{(k)}\right.\right. \\
& \left.+\tilde{B}_{2}^{(j) *} \tilde{B}_{4}^{(k)}+3 \tilde{B}_{1}^{(j)} \tilde{B}_{5}^{(k) *}+\tilde{B}_{1}^{(j) *} \tilde{B}_{5}^{(k) *}+3 \tilde{B}_{2}^{(j)} \tilde{B}_{5}^{(k) *}\right) \\
& +\tilde{A}_{2}^{(i)}\left\{\frac{1}{4}\left(\tilde{B}_{1}^{(j)} \tilde{B}_{1}^{(k)}+2 \tilde{B}_{1}^{(j)} \tilde{B}_{1}^{(k) *}+2 \tilde{B}_{1}^{(j) *} \tilde{B}_{2}^{(k)}+2 \tilde{B}_{2}^{(j)} \tilde{B}_{2}^{(k) *}\right)+4\left(\tilde{B}_{1}^{(j)} \tilde{B}_{4}^{(k)}+\tilde{B}_{1}^{(j) *} \tilde{B}_{4}^{(k)}-\tilde{B}_{1}^{(j)} \tilde{B}_{5}^{(k) *}-\tilde{B}_{2}^{(j)} \tilde{B}_{5}^{(k) *}\right)\right\} \\
& \left.-2 \tilde{A}_{1}^{(i)}\left(3 \tilde{C}_{8}^{(j k)}+\tilde{C}_{9}^{(j k) *}+2 \tilde{C}_{12}^{(j k)}\right)+2 \tilde{A}_{2}^{(i)}\left(\tilde{C}_{1}^{(j k)}+4 \tilde{C}_{6}^{(j k)}+4 \tilde{C}_{7}^{(j k)}+4 \tilde{C}_{8}^{(j k)}+4 \tilde{C}_{10}^{(j k)}\right)\right]_{s^{\prime}} e^{i \Psi_{x}\left(s^{\prime}, s\right)+i 2 \Psi_{y}\left(s^{\prime}, s\right)},
\end{aligned}
$$




$$
\begin{aligned}
\tilde{D}_{9}^{(i j k)}(s) \equiv & \frac{1}{2 \sin \pi\left(\nu_{x}-2 \nu_{y}\right)} \int_{s}^{s+C} d s^{\prime}\left[-\frac{1}{2} \tilde{A}_{1}^{(i)}\left(\tilde{B}_{1}^{(j) *} \tilde{B}_{4}^{(k) *}+3 \tilde{B}_{1}^{(j)} \tilde{B}_{4}^{(k) *}+3 \tilde{B}_{2}^{(j)} \tilde{B}_{4}^{(k) *}+3 \tilde{B}_{1}^{(j) *} \tilde{B}_{5}^{(k)}+3 \tilde{B}_{1}^{(j)} \tilde{B}_{5}^{(k)}+\tilde{B}_{2}^{(j) *} \tilde{B}_{5}^{(k)}\right.\right. \\
& \left.+\tilde{B}_{2}^{(j)} \tilde{B}_{5}^{(k)}\right)+\tilde{A}_{2}^{(i)}\left\{\frac{1}{4}\left(2 \tilde{B}_{1}^{(j)} \tilde{B}_{1}^{(k) *}+\tilde{B}_{1}^{(j)} \tilde{B}_{1}^{(k)}+2 \tilde{B}_{1}^{(j) *} \tilde{B}_{2}^{(k)}+2 \tilde{B}_{2}^{(j)} \tilde{B}_{2}^{(k) *}\right)\right. \\
& \left.+4\left(\tilde{B}_{1}^{(j)} \tilde{B}_{4}^{(k) *}+\tilde{B}_{2}^{(j)} \tilde{B}_{4}^{(k) *}-\tilde{B}_{1}^{(j) *} \tilde{B}_{5}^{(k)}-\tilde{B}_{1}^{(j)} \tilde{B}_{5}^{(k)}\right)\right\}-2 \tilde{A}_{1}^{(i)}\left(\tilde{C}_{8}^{(j k) *}+3 \tilde{C}_{9}^{(j k)}+2 \tilde{C}_{13}^{(j k)}\right) \\
& \left.+2 \tilde{A}_{2}^{(i)}\left(\tilde{C}_{1}^{(j k)}+4 \tilde{C}_{6}^{(j k) *}+4 \tilde{C}_{7}^{(j k) *}-4 \tilde{C}_{9}^{(j k)}-4 \tilde{C}_{11}^{(j k)}\right)\right]_{s^{\prime}} e^{i \Psi_{x}\left(s^{\prime}, s\right)-i 2 \Psi_{y}\left(s^{\prime}, s\right)}
\end{aligned}
$$

From Eqs. (52), (53) and (54), we then have

$$
\begin{aligned}
F_{\alpha \beta}^{(i j)} & =\frac{1}{2 \pi} \int_{0}^{C} d s \tilde{W}_{\alpha \beta}^{(i j)}(s), \quad(\alpha \beta=x x, x y, y y), \\
\tilde{W}_{x x}^{(i j)}(s) & =\operatorname{Re}\left[-2 \tilde{A}_{1}^{(i)}\left(3 \tilde{B}_{1}^{(j)}+\tilde{B}_{2}^{(j)}\right)\right]_{s}, \\
\tilde{W}_{x y}^{(i j)}(s) & =\operatorname{Re}\left[12 \tilde{A}_{1}^{(i)} \tilde{B}_{3}^{(j)}+4 \tilde{A}_{2}^{(i)}\left(\tilde{B}_{1}^{(j)}-2 \tilde{B}_{4}^{(j)}+2 \tilde{B}_{5}^{(j)}\right)\right]_{s}, \\
\tilde{W}_{y y}^{(i j)}(s) & =\operatorname{Re}\left[-2 \tilde{A}_{2}^{(i)}\left(4 \tilde{B}_{3}^{(j)}+\tilde{B}_{4}^{(j)}+\tilde{B}_{5}^{(j)}\right)\right]_{s},
\end{aligned}
$$

for the second order coefficients, and from Eqs. (80) and (81), we have

$$
\begin{aligned}
& F_{x x x}^{(i j k l)}=\frac{1}{2 \pi} \int_{0}^{C} d s \tilde{W}_{x x x}^{(i j k l)}(s), \\
& F_{x x y}^{(i j k l)}=\frac{1}{2 \pi} \int_{0}^{C} d s \tilde{W}_{x x y}^{(i j k l)}(s),
\end{aligned}
$$

$$
\begin{aligned}
\tilde{W}_{x x x}^{(i j k l)}(s)= & 2 \operatorname{Re} \tilde{A}_{1}^{(i)}(s)\left[\frac{1}{24}\left(\tilde{B}_{1}^{(j)} \tilde{B}_{1}^{(k)} \tilde{B}_{1}^{(l)}+9 \tilde{B}_{1}^{(j)} \tilde{B}_{1}^{(k)} \tilde{B}_{1}^{(l) *}+6 \tilde{B}_{1}^{(j)} \tilde{B}_{1}^{(k) *} \tilde{B}_{2}^{(l)}+9 \tilde{B}_{1}^{(j)} \tilde{B}_{1}^{(k)} \tilde{B}_{2}^{(l) *}+18 \tilde{B}_{1}^{(j)} \tilde{B}_{2}^{(k)} \tilde{B}_{2}^{(l) *}+3 \tilde{B}_{2}^{(j)} \tilde{B}_{2}^{(k)} \tilde{B}_{2}^{(l) *}\right)\right. \\
& \left.+\left(\tilde{B}_{1}^{(j)}+3 \tilde{B}_{1}^{(j) *}+3 \tilde{B}_{2}^{(j) *}\right) \tilde{C}_{1}^{(k l)}+2\left(\tilde{B}_{1}^{(j) *}+3 \tilde{B}_{2}^{(j) *}\right) \tilde{C}_{2}^{(k l)}+3 \tilde{B}_{2}^{(j) *} \tilde{C}_{3}^{(k l)}-3 \tilde{D}_{1}^{(j k l)}-3 \tilde{D}_{2}^{(j k l)}\right]_{s} \\
\tilde{W}_{x x y}^{(i j k l)}(s)= & 2 \operatorname{Re} \tilde{A}_{1}^{(i)}(s)\left[-\frac{1}{4}\left(\tilde{B}_{1}^{(j)} \tilde{B}_{1}^{(k)} \tilde{B}_{3}^{(l)}+3 \tilde{B}_{1}^{(j)} \tilde{B}_{1}^{(k)} \tilde{B}_{3}^{(l) *}+6 \tilde{B}_{1}^{(j)} \tilde{B}_{1}^{(k) *} \tilde{B}_{3}^{(l)}+2 \tilde{B}_{1}^{(j)} \tilde{B}_{2}^{(k) *} \tilde{B}_{3}^{(l) *}+2 \tilde{B}_{1}^{(j)} \tilde{B}_{2}^{(k)} \tilde{B}_{3}^{(l) *}\right.\right. \\
& \left.+6 \tilde{B}_{1}^{(j)} \tilde{B}_{2}^{(k) *} \tilde{B}_{3}^{(l)}+6 \tilde{B}_{2}^{(j)} \tilde{B}_{2}^{(k) *} \tilde{B}_{3}^{(l)}\right)-2\left(\tilde{B}_{3}^{(j)}+3 \tilde{B}_{3}^{(j) *}\right) \tilde{C}_{1}^{(k l)}-4 \tilde{B}_{3}^{(j) *} \tilde{C}_{2}^{(k l)}+\left(\tilde{B}_{1}^{(j)}+3 \tilde{B}_{1}^{(j) *}+3 \tilde{B}_{2}^{(j) *}\right) \tilde{C}_{4}^{(k l)} \\
& \left.+2\left(\tilde{B}_{1}^{(j) *}+3 \tilde{B}_{2}^{(j) *}\right) \tilde{C}_{5}^{(k l)}-3\left(\tilde{D}_{6}^{(j k l)}+\tilde{D}_{7}^{(j k l)}\right)\right]_{s}+2 \operatorname{Re} \tilde{A}_{2}^{(i)}(s)\left[\frac{3}{4}\left(\tilde{B}_{1}^{(j)} \tilde{B}_{1}^{(k)} \tilde{B}_{1}^{(l) *}+\tilde{B}_{1}^{(j)} \tilde{B}_{1}^{(k)} \tilde{B}_{2}^{(l) *}+2 \tilde{B}_{1}^{(j)} \tilde{B}_{2}^{(k)} \tilde{B}_{2}^{(l) *}\right)\right. \\
& +\frac{1}{2}\left(2 \tilde{B}_{1}^{(j)} \tilde{B}_{1}^{(k) *} \tilde{B}_{4}^{(l)}-2 \tilde{B}_{1}^{(j)} \tilde{B}_{1}^{(k) *} \tilde{B}_{5}^{(l)}+2 \tilde{B}_{1}^{(j)} \tilde{B}_{2}^{(k) * *} \tilde{B}_{4}^{(l)}-2 \tilde{B}_{1}^{(j)} \tilde{B}_{2}^{(k) *} \tilde{B}_{5}^{(l)}\right. \\
& \left.+\tilde{B}_{1}^{(j)} \tilde{B}_{1}^{(k)} \tilde{B}_{4}^{(l) *}-\tilde{B}_{1}^{(j)} \tilde{B}_{1}^{(k)} \tilde{B}_{5}^{(l) *}+2 \tilde{B}_{2}^{(j)} \tilde{B}_{2}^{(k) *} \tilde{B}_{4}^{(l)}-2 \tilde{B}_{2}^{(j)} \tilde{B}_{2}^{(k) *} \tilde{B}_{5}^{(l)}\right)+2\left(\tilde{B}_{1}^{(j) *}+\tilde{B}_{2}^{(j) *}+2 \tilde{B}_{4}^{(j) *}-2 \tilde{B}_{5}^{(j) *}\right) \tilde{C}_{1}^{(k l)} \\
& +4 \tilde{B}_{2}^{(j) *} \tilde{C}_{2}^{(k l)}+2\left(-\left(\tilde{B}_{1}^{(j)}+\tilde{B}_{1}^{(j) *}\right) \tilde{C}_{6}^{(k l)}-\left(\tilde{B}_{1}^{(j) *}+\tilde{B}_{2}^{(j) *}\right) \tilde{C}_{8}^{(k l)}+\left(\tilde{B}_{1}^{(j) *}+\tilde{B}_{2}^{(j) *}\right) \tilde{C}_{9}^{(k l)}-\tilde{B}_{2}^{(j) *} \tilde{C}_{12}^{(k l)}+\tilde{B}_{2}^{(j) *} \tilde{C}_{13}^{(k l)}\right) \\
& \left.+2\left(\tilde{D}_{1}^{(j k l)}+2 \tilde{D}_{8}^{(j k l)}-2 \tilde{D}_{9}^{(j k l)}\right)\right]_{s} \\
&
\end{aligned}
$$


for the fourth-order coefficients. Once $\tilde{A}_{1}^{(i)}(s)$ and $\tilde{A}_{2}^{(i)}(s)$ are given as Eqs. (93) and (94), $\tilde{B}_{n}^{(i)}(s), \tilde{C}_{n}^{(i)}(s)$, and $\tilde{D}_{n}^{(i)}(s)$ are obtained by carrying out integrals using Eqs. (C4), (C5), and (C6) in turn, and the coefficients $F_{\alpha \beta}^{(i j)}, F_{x x x}^{(i j k l)}$, and $F_{x x y}^{(i j k l)}$ are finally evaluated by integrating $\tilde{W}_{\alpha \beta}^{(i j)}(s)$, $\tilde{W}_{x x x}^{(i j k l)}(s)$, and $\tilde{W}_{x x y}^{(i j k l)}(s)$. We note that all of these integrals contain the step function $\Delta_{i}(s)$ defined by Eq. (95) in their integrand. This means that in numerical calculations, we need to know the integrand only at sextupole magnet positions $s_{i}^{(m)}$, i.e., the position on the $m$ th mesh point in the $i$ th sextupole magnet.

[1] E. A. Crosbie, Improvement of the dynamic aperture in Chasman Green lattice design light source storage rings, in 1987 Particle Accelerator Conference (IEEE, Washington, DC, 1987), p. 443.

[2] S. Y. Lee, Accelerator Physics (World Scientific, Singapore, 2004), Chap. 2, Section VII.3.

[3] See, for example, P. Raimondi, The ESRF low emittance upgrade, in Proceedings of IPAC2016, p. 2023 for ESRFEBS, http://accelconf.web.cern.ch/AccelConf/ipac2016/ papers/wexa01.pdf; S. Henderson, Status of the APS upgrade project, in Proceedings of IPAC2015, p. 1791 for APS-U, http://accelconf.web.cern.ch/AccelConf/IPAC2015/papers/ tupje067.pdf; C. Steier et al., Physics design progress towards a diffraction limited upgrade of the ALS, in Proceedings of IPAC2016, p. 2956 for ALS-U, http://accelconf.web.cern.ch/ AccelConf/ipac2016/papers/wepow049.pdf; M. Eriksson, Commissioning of the MAX IV light source, in Proceedings of IPAC2016, p. 11 for MAX IV, http://accelconf .web.cern.ch/AccelConf/ipac2016/papers/moyaa01.pdf L. Liu et al., A new optics for SIRIUS, in Proceedings of IPAC2016, p. 3413 for Sirius, http://accelconf.web.cern.ch/ AccelConf/ipac2016/papers/thpmr013.pdf.
[4] H. Tanaka et al., SPring-8 upgrade project, in Proceedings of IPAC2016, p. 2867, http://accelconf.web.cern.ch/ AccelConf/ipac2016/papers/wepow019.pdf.

[5] K. Soutome et al., Non-linear optimization of storage ring lattice for the SPring-8 upgrade, in Proceedings of IPAC2016, p. 3440, http://accelconf.web.cern.ch/AccelConf/ipac2016/ papers/thpmr022.pdf.

[6] J. Irwin and A. Dragt, in Handbook of Accelerator Physics, edited by A. W. Chao and M. Tigner (World Scientific, Singapore, 2006), Section 2.3.5.

[7] A. Dragt, in [6], Section 2.3.6.

[8] M. Berz, in [6], Section 2.3.7.

[9] M. Berz, Modern Map Methods in Particle Beam Physics (Academic Press, New York, 1999).

[10] É. Forest, Beam Dynamics: A New Attitude and Framework (Taylor \& Francis, New York, 1998).

[11] M. Berz, High-order computation and normal form analysis of repetitive systems, AIP Conf. Proc. 249, 456 (1992).

[12] Although the importance of higher-order terms in conjunction with phase space trajectories near the resonances has been reported in e.g. R. Nagaoka et al., Nonlinear dynamics with sextupoles in low-emittance light source storage rings, Nucl. Instrum. Methods Phys. Res., Sect. A 302, 9 (1991) and F. Schmidt and F. Willeke, Nonlinear beam dynamics close to resonances excited by sextupole fields, in Proceedings of EPAC1988, p. 908, discussions are limited to one-dimensional calculations in the horizontal plane, http://accelconf.web.cern.ch/AccelConf/e88/ PDF/EPAC1988_0908.PDF.

[13] R. D. Ruth, Single-particle dynamics in circular accelerators, AIP Conf. Proc. 153, 150 (1987).

[14] A. J. Lichtenberg and M. A. Lieberman, Regular and Chaotic Dynamics, Applied Mathematical Sciences Vol. 38 (Springer-Verlag, New York, 1983).

[15] M. Takao (unpublished); M. Takao, Formulation of nonlinear chromaticity in circular accelerators by canonical perturbation method, Phys. Rev. E 72, 046502 (2005).

[16] L. Michelotti, Introduction to the nonlinear dynamics arising from magnetic multipoles, AIP Conf. Proc. 153, 236 (1987). 